\title{
Influence of Sodium Cationization versus Protonation on the Gas-Phase Conformations and Glycosidic Bond Stabilities of 2'-Deoxyadenosine and Adenosine
}

\author{
Y. Zhu ${ }^{\dagger}$, L. A. Hamlow ${ }^{\dagger}$, C. C. He ${ }^{\dagger}$, S. F. Strobehn ${ }^{\dagger}$, J. K. Lee ${ }^{\dagger}, J_{.}$Gao $^{\ddagger}$, G. Berden $^{\ddagger}$, \\ J. Oomens ${ }^{*}$, and M. T. Rodgers ${ }^{\dagger} *$ \\ tDepartment of Chemistry, Wayne State University, Detroit, MI, 48202 \\ ${ }^{7}$ Radboud University, Institute for Molecules and Materials, FELIX Laboratory, Toernooiveld 7c, \\ 6525ED Nijmegen, The Netherlands
}

Full citation for Gaussian09, reference 61:

Frisch, M. J.; Trucks, G. W.; Schlegel, H. B.; Scuseria, G. E.; Robb, M. A.; Cheeseman, J. R.; Scalmani, G.; Barone, V.; Mennucci, B.; Petersson, G. A.; Nakatsuji, H.; Caricato, M.; Li, X.; Hratchian, H. P.; Izmaylov,A. F.; Bloino, J.; Zheng, G.; Sonnenberg, J. L.; Hada, M.; Ehara, M.; Toyota, K.; Fukuda, R.; Hasegawa, J.; Ishida, M.; Nakajima, T.; Honda, Y.; Kitao, O.; Nakai, H.; Vreven, T.; Montgomery Jr., J. A.; Peralta, J. E.; Ogliaro, F.; Bearpark, M. J.; Heyd, J.; Brothers, E. N.; Kudin, K. N.; Staroverov, V. N.; Kobayashi, R.; Normand, J.; Raghavachari, K.; Rendell, A. P.; Burant, J. C.; Iyengar, S. S.; Tomasi, J.; Cossi, M.; Rega, N.; Millam, N. J.; Klene, M.; Knox, J. E.; Cross, J. B.; Bakken, V.; Adamo, C.; Jaramillo, J.; Gomperts, R.; Stratmann, R. E.; Yazyev, O.; Austin, A. J.; Cammi, R.; Pomelli, C.; Ochterski, J. W.; Martin, R. L.; Morokuma, K.; Zakrzewski, V. G.; Voth, G. A.; Salvador, P.; Dannenberg, J. J.; Dapprich, S.; Daniels, A. D.; Farkas, Ö.; Foresman, J. B.; Ortiz, J. V.; Cioslowski, J.; Fox, D. J. Gaussian 09, Gaussian, Inc.: Wallingford, CT, USA, 2009. 
Table S1. Relative Enthalpies and Free Energies of All Stable Low-Energy Conformers of $[\mathrm{dAdo}+\mathrm{Na}]^{+}$and $[\mathrm{Ado}+\mathrm{Na}]^{+}$at 0 and $298 \mathrm{~K}$ in kJ/mol ${ }^{\alpha}$

\begin{tabular}{|c|c|c|c|c|c|c|c|}
\hline Species & Conformer & $\Delta \mathrm{H}_{0}$ & $\Delta \mathrm{H}_{298}$ & $\Delta \mathrm{G}_{298}$ & $\begin{array}{c}\text { Adenine } \\
\text { Orientation }\end{array}$ & $\mathrm{P}$ & Sugar Puckering \\
\hline \multirow[t]{29}{*}[\mathrm{dAdo}+\mathrm{Na}]{$^{+}$} & $\mathrm{T} 1\left(\mathrm{~N} 3 \mathrm{O} 4{ }^{\prime} \mathrm{O} 5 '\right)$ & 4.1 & 5.1 & 0.0 & syn & $144.6^{\circ}$ & C1'-exo $\left({ }_{1}^{2} \mathrm{~T}\right)$ \\
\hline & T2(N3O4'O5') & 0.0 & 0.0 & 1.4 & syn & $103.3^{\circ}$ & $\mathrm{O} 4$ '-endo $\left({ }^{\mathrm{O}} \mathrm{T}_{4}\right)$ \\
\hline & T3(N3O4'O5') & 0.0 & 0.0 & 1.5 & syn & $103.4^{\circ}$ & O4'-endo $\left({ }^{\mathrm{O}} \mathrm{T}_{4}\right)$ \\
\hline & T4(N3O4'O5') & 4.3 & 5.2 & 2.2 & syn & $147.4^{\circ}$ & $\mathrm{C} 2$ '-endo $\left({ }^{2} \mathrm{~T}_{1}\right)$ \\
\hline & T5(N3O4'O5') & 2.2 & 2.5 & 3.5 & syn & $344.9^{\circ}$ & $C 2^{\prime}-\operatorname{exo}\left({ }_{2} T^{3}\right)$ \\
\hline & T6(N3O4'O5') & 8.3 & 8.4 & 9.9 & syn & $345.7^{\circ}$ & $C 2^{\prime}$-exo $\left({ }_{2} T^{3}\right)$ \\
\hline & T7(N3O4'O5') & 9.0 & 9.3 & 10.3 & syn & $152.6^{\circ}$ & C2'-endo $\left({ }^{2} \mathrm{~T}_{1}\right)$ \\
\hline & T8(N3O4'O5') & 20.5 & 21.2 & 20.4 & syn & $141.5^{\circ}$ & $\mathrm{C} 1^{\prime}-\operatorname{exo}\left({ }_{1} \mathrm{~T}^{2}\right)$ \\
\hline & T9(N3O4'O5') & 28.2 & 28.3 & 29.5 & syn & $9.7^{\circ}$ & C3'-endo $\left({ }^{3} \mathrm{~T}_{2}\right)$ \\
\hline & B1(N3O4') & 35.2 & 36.5 & 33.7 & syn & $193.5^{\circ}$ & C3'-exo $\left({ }_{3} \mathrm{~T}^{2}\right)$ \\
\hline & $\mathrm{B} 1\left(\mathrm{O} 4{ }^{\prime} \mathrm{O} 5 '\right)$ & 48.0 & 49.6 & 41.4 & syn & $14.5^{\circ}$ & C3'-endo $\left({ }^{3} \mathrm{~T}_{2}\right)$ \\
\hline & B2(N3O4') & 50.4 & 51.9 & 48.3 & syn & $205.5^{\circ}$ & C3'-exo $\left({ }_{3} \mathrm{~T}^{4}\right)$ \\
\hline & $\mathrm{B} 1\left(\mathrm{~N} \mathrm{NH}_{2}\right)$ & 66.2 & 66.6 & 65.0 & anti & $173.2^{\circ}$ & $\mathrm{C} 2$ '-endo $\left({ }^{2} \mathrm{~T}_{3}\right)$ \\
\hline & B1(N3O3') & 66.3 & 67.1 & 66.4 & anti & $138.1^{\circ}$ & $\mathrm{C} 1^{\prime}-\operatorname{exo}\left({ }_{1} \mathrm{~T}^{2}\right)$ \\
\hline & $\mathrm{B} 2\left(\mathrm{~N} \mathrm{NH}_{2}\right)$ & 69.8 & 70.2 & 69.3 & anti & $9.4^{\circ}$ & C3'-endo $\left({ }^{3} \mathrm{~T}_{2}\right)$ \\
\hline & $\mathrm{B} 3\left(\mathrm{~N} \mathrm{NH} \mathrm{N}_{2}\right)$ & 74.1 & 74.7 & 72.5 & anti & $179.6^{\circ}$ & $C 2$ '-endo $\left({ }^{2} \mathrm{~T}_{3}\right)$ \\
\hline & B1(N7O5') & 73.0 & 73.2 & 72.6 & anti & $293.5^{\circ}$ & C1'-endo $\left({ }^{1} \mathrm{~T}_{4}\right)$ \\
\hline & $\mathrm{B} 1\left(\mathrm{~N} 1 \mathrm{NH}_{2}\right)$ & 74.5 & 75.0 & 73.1 & $a n t i$ & $173.3^{\circ}$ & $\mathrm{C} 2^{\prime}$-endo $\left({ }^{2} \mathrm{~T}_{3}\right)$ \\
\hline & $\mathrm{B} 4\left(\mathrm{~N} \mathrm{NH} \mathrm{H}_{2}\right)$ & 78.4 & 79.6 & 74.1 & anti & $155.0^{\circ}$ & $\mathrm{C} 2$ '-endo $\left({ }^{2} \mathrm{~T}_{1}\right)$ \\
\hline & $\mathrm{B} 2\left(\mathrm{~N} 1 \mathrm{NH}_{2}\right)$ & 77.4 & 78.4 & 74.4 & anti & $10.4^{\mathrm{o}}$ & C3'-endo $\left({ }^{3} \mathrm{~T}_{2}\right)$ \\
\hline & $\mathrm{B} 5\left(\mathrm{~N}_{7} \mathrm{NH}_{2}\right)$ & 79.7 & 81.0 & 74.8 & anti & $153.9^{\circ}$ & $\mathrm{C} 2$ '-endo $\left({ }^{2} \mathrm{~T}_{1}\right)$ \\
\hline & $\mathrm{B} 6\left(\mathrm{~N} \mathrm{NH}_{2}\right)$ & 77.6 & 78.2 & 75.5 & anti & $201.7^{\circ}$ & C3'-exo $\left({ }_{3} \mathrm{~T}^{4}\right)$ \\
\hline & $\mathrm{B} 7\left(\mathrm{~N} \mathrm{NH} \mathrm{N}_{2}\right)$ & 79.5 & 80.6 & 75.7 & syn & $164.5^{\circ}$ & $\mathrm{C} 2^{\prime}$-endo $\left({ }^{2} \mathrm{~T}_{3}\right)$ \\
\hline & $\mathrm{B} 8\left(\mathrm{~N}_{7 \mathrm{NH}_{2}}\right)$ & 80.8 & 82.3 & 75.8 & anti & $157.8^{\circ}$ & C2'-endo $\left({ }^{2} \mathrm{~T}_{1}\right)$ \\
\hline & $\mathrm{B} 9\left(\mathrm{~N}_{7 \mathrm{NH}_{2}}\right)$ & 78.4 & 79.1 & 76.0 & anti & $202.2^{\circ}$ & C3'-exo $\left({ }_{3} T^{4}\right)$ \\
\hline & $\mathrm{B} 10\left(\mathrm{~N} \mathrm{NH}_{2}\right)$ & 79.4 & 80.3 & 76.8 & anti & $200.0^{\circ}$ & C3'-exo $\left({ }_{3} \mathrm{~T}^{4}\right)$ \\
\hline & $\mathrm{B} 11\left(\mathrm{~N} \mathrm{NH}_{2}\right)$ & 81.0 & 81.7 & 77.4 & syn & $41.6^{\circ}$ & C4'-exo $\left({ }_{4} \mathrm{~T}^{3}\right)$ \\
\hline & $\mathrm{B} 3\left(\mathrm{~N} 1 \mathrm{NH}_{2}\right)$ & 85.8 & 87.3 & 77.6 & anti & $147.1^{\circ}$ & $\mathrm{C} 2$ '-endo $\left({ }^{2} \mathrm{~T}_{1}\right)$ \\
\hline & $\mathrm{B} 12\left(\mathrm{~N} \mathrm{NH}_{2}\right)$ & 81.3 & 82.1 & 77.9 & syn & $41.5^{\circ}$ & C4'-exo $\left({ }_{4} \mathrm{~T}^{3}\right)$ \\
\hline
\end{tabular}


Table S1. Relative Enthalpies and Free Energies of All Stable Low-Energy Conformers of $[\mathrm{dAdo}+\mathrm{Na}]^{+}$and $[\mathrm{Ado}+\mathrm{Na}]^{+}$at 0 and $298 \mathrm{~K}$ in $\mathrm{kJ} / \mathrm{mol}^{\alpha}$

\begin{tabular}{|c|c|c|c|c|c|c|c|}
\hline Species & Conformer & $\Delta \mathrm{H}_{0}$ & $\Delta \mathrm{H}_{298}$ & $\Delta \mathrm{G}_{298}$ & $\begin{array}{c}\text { Adenine } \\
\text { Orientation }\end{array}$ & $\mathrm{P}$ & Sugar Puckering \\
\hline \multirow[t]{19}{*}{ 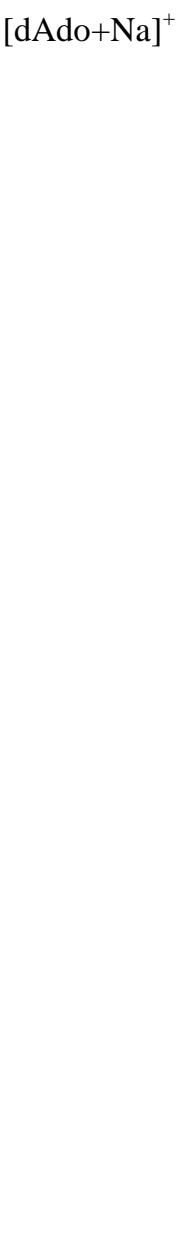 } & $\mathrm{B} 4\left(\mathrm{~N} 1 \mathrm{NH}_{2}\right)$ & 86.8 & 87.9 & 79.0 & anti & $43.0^{\circ}$ & $\mathrm{C} 4^{\prime}-\operatorname{exo}\left({ }_{4} \mathrm{~T}^{3}\right)$ \\
\hline & B1(N3O5') & 79.4 & 79.4 & 79.5 & anti & $289.2^{\circ}$ & $\mathrm{C} 1^{\prime}$-endo $\left({ }^{1} \mathrm{~T}\right)$ \\
\hline & $\mathrm{B} 5\left(\mathrm{~N} 1 \mathrm{NH}_{2}\right)$ & 89.1 & 91.1 & 81.2 & anti & $152.6^{\circ}$ & $\mathrm{C} 2$ '-endo $\left({ }^{2} \mathrm{~T}_{1}\right)$ \\
\hline & $\mathrm{B} 13\left(\mathrm{~N} \mathrm{NH}_{2}\right)$ & 86.3 & 87.1 & 82.4 & syn & $162.3^{\circ}$ & $C 2^{\prime}$-endo $\left({ }^{2} \mathrm{E}\right)$ \\
\hline & $\mathrm{B} 14\left(\mathrm{~N} \mathrm{NH}_{2}\right)$ & 87.1 & 88.3 & 83.1 & syn & $39.6^{\circ}$ & C4'-exo $\left({ }_{4} \mathrm{~T}^{3}\right)$ \\
\hline & $\mathrm{T} 1\left(\mathrm{~N} \mathrm{NH}_{2} \mathrm{O} 5^{\prime}\right)$ & 81.6 & 80.7 & 85.2 & anti & $339.5^{\circ}$ & $\mathrm{C} 2^{\prime}-\operatorname{exo}\left({ }_{2} \mathrm{~T}^{1}\right)$ \\
\hline & $\mathrm{B} 15\left(\mathrm{~N} \mathrm{NH}_{2}\right)$ & 93.7 & 95.2 & 87.3 & anti & $173.8^{\circ}$ & $\mathrm{C} 2$ '-endo $\left({ }^{2} \mathrm{~T}_{3}\right)$ \\
\hline & $\mathrm{B} 6\left(\mathrm{~N}_{1} \mathrm{NH}_{2}\right)$ & 90.7 & 91.5 & 87.7 & anti & $199.9^{\circ}$ & $\mathrm{C} 3$ '-exo $\left({ }_{3} \mathrm{~T}^{4}\right)$ \\
\hline & $\mathrm{B} 7\left(\mathrm{~N}_{1} \mathrm{NH}_{2}\right)$ & 91.3 & 92.1 & 88.3 & anti & $199.8^{\circ}$ & $\mathrm{C} 3$ '-exo $\left({ }_{3} \mathrm{E}\right)$ \\
\hline & $\mathrm{B} 8\left(\mathrm{~N}_{1} \mathrm{NH}_{2}\right)$ & 91.4 & 92.0 & 88.4 & syn & $171.0^{\circ}$ & $\mathrm{C} 2$ '-endo $\left({ }^{2} \mathrm{~T}_{1}\right)$ \\
\hline & $\mathrm{B} 16\left(\mathrm{~N} \mathrm{NH}_{2}\right)$ & 91.7 & 92.2 & 88.6 & anti & $22.5^{\circ}$ & C3'-endo $\left({ }^{3} \mathrm{~T}_{4}\right)$ \\
\hline & $\mathrm{B} 9\left(\mathrm{~N}_{1} \mathrm{NH}_{2}\right)$ & 91.7 & 92.5 & 89.0 & anti & $199.8^{\circ}$ & $\mathrm{C} 3^{\prime}-\operatorname{exo}\left({ }_{3} \mathrm{~T}^{4}\right)$ \\
\hline & $\mathrm{T} 2\left(\mathrm{~N} \mathrm{NH}_{2} \mathrm{O}^{\prime}\right)$ & 86.8 & 85.9 & 90.3 & anti & $340.8^{\circ}$ & $\mathrm{C} 2^{\prime}-\operatorname{exo}\left({ }_{2} \mathrm{~T}^{1}\right)$ \\
\hline & $\mathrm{T} 3\left(\mathrm{~N} \mathrm{NH}_{2} \mathrm{O} 5^{\prime}\right)$ & 89.6 & 89.1 & 90.9 & syn & $250.5^{\circ}$ & C4'-endo $\left({ }^{4} \mathrm{~T}_{\mathrm{O}}\right)$ \\
\hline & $\mathrm{B} 10\left(\mathrm{~N} 1 \mathrm{NH}_{2}\right)$ & 95.9 & 97.3 & 91.2 & syn & $166.4^{\circ}$ & $\mathrm{C} 2$ '-endo $\left({ }^{2} \mathrm{~T}_{3}\right)$ \\
\hline & $\mathrm{B} 17\left(\mathrm{~N} \mathrm{NH}_{2}\right)$ & 94.9 & 96.2 & 91.6 & syn & $184.5^{\circ}$ & $\mathrm{C} 3^{\prime}-\operatorname{exo}\left({ }_{3} \mathrm{~T}^{2}\right)$ \\
\hline & $\mathrm{B} 11\left(\mathrm{~N} 1 \mathrm{NH}_{2}\right)$ & 100.5 & 102.7 & 93.0 & anti & $179.2^{\circ}$ & $\mathrm{C} 2$ '-endo $\left({ }^{2} \mathrm{~T}_{3}\right)$ \\
\hline & M1(N3) & 100.9 & 102.7 & 95.4 & anti & $9.1^{\circ}$ & $\mathrm{C} 3^{\prime}$-endo $\left({ }^{3} \mathrm{~T}_{2}\right)$ \\
\hline & M2(N3) & 107.0 & 109.3 & 99.9 & anti & $55.0^{\circ}$ & $\mathrm{C} 4$ '-exo $\left({ }_{4} \mathrm{E}\right)$ \\
\hline \multirow[t]{10}{*}[\mathrm{Ado}+\mathrm{Na}]{$^{+}$} & T1(N3O4'O5') & 0.0 & 0.0 & 0.0 & syn & $113.5^{\circ}$ & $\mathrm{C} 1^{\prime}-\operatorname{exo}\left({ }_{1} \mathrm{~T}^{\mathrm{O}}\right)$ \\
\hline & T2(N3O4'O5') & 0.5 & 0.1 & 1.0 & syn & $78.1^{\circ}$ & O4'-endo $\left({ }^{\mathrm{O}} \mathrm{T}_{4}\right)$ \\
\hline & T3(N3O4'O5') & 5.2 & 5.4 & 4.3 & syn & $139.2^{\circ}$ & $\mathrm{C} 1^{\prime}-\operatorname{exo}\left({ }_{1} \mathrm{~T}^{2}\right)$ \\
\hline & B1(N3O2') & 20.3 & 19.8 & 19.7 & anti & $68.1^{\circ}$ & $C 4^{\prime}-\operatorname{exo}\left({ }_{4} \mathrm{~T}^{\mathrm{O}}\right)$ \\
\hline & B2(N3O2') & 22.1 & 21.6 & 21.2 & anti & $204.6^{\circ}$ & C3'-exo $\left({ }_{3} \mathrm{~T}^{4}\right)$ \\
\hline & $\mathrm{B} 3\left(\mathrm{~N} 3 \mathrm{O} 2{ }^{\prime}\right)$ & 23.7 & 24.0 & 21.3 & anti & $76.0^{\circ}$ & $\mathrm{O} 4$ '-endo $\left({ }^{\mathrm{O}} \mathrm{T}_{4}\right)$ \\
\hline & $\mathrm{B} 4\left(\mathrm{~N} 3 \mathrm{O} 2{ }^{\prime}\right)$ & 31.4 & 31.4 & 27.9 & anti & $198.4^{\circ}$ & $\mathrm{C} 3$ '-exo $\left({ }_{3} \mathrm{E}\right)$ \\
\hline & $\mathrm{B} 5\left(\mathrm{~N} 3 \mathrm{O} 2{ }^{\prime}\right)$ & 32.5 & 32.7 & 30.2 & anti & $75.4^{\circ}$ & $\mathrm{O} 4$ '-endo $\left({ }^{\mathrm{O}} \mathrm{T}_{4}\right)$ \\
\hline & B6(N3O2') & 36.2 & 36.7 & 33.2 & anti & $76.0^{\circ}$ & O4'-endo $\left({ }^{\mathrm{O}} \mathrm{T}_{4}\right)$ \\
\hline & $\mathrm{T} 1\left(\mathrm{~N} 3 \mathrm{O} 2{ }^{\prime} \mathrm{O} 3{ }^{\prime}\right)$ & 36.4 & 37.3 & 36.0 & anti & $140.4^{\circ}$ & $\mathrm{C} 1^{\prime}$-exo $\left({ }_{1} \mathrm{~T}^{2}\right)$ \\
\hline
\end{tabular}


Table S1. Relative Enthalpies and Free Energies of All Stable Low-Energy Conformers of $[\mathrm{dAdo}+\mathrm{Na}]^{+}$and $[\mathrm{Ado}+\mathrm{Na}]^{+}$at 0 and $298 \mathrm{~K}$ in kJ/mol ${ }^{\alpha}$

\begin{tabular}{|c|c|c|c|c|c|c|c|}
\hline Species & Conformer & $\Delta \mathrm{H}_{0}$ & $\Delta \mathrm{H}_{298}$ & $\Delta \mathrm{G}_{298}$ & $\begin{array}{c}\text { Adenine } \\
\text { Orientation }\end{array}$ & $\mathrm{P}$ & Sugar Puckering \\
\hline \multirow[t]{29}{*}[\mathrm{Ado}+\mathrm{Na}]{$^{+}$} & B1(N3O4') & 42.3 & 43.0 & 39.5 & syn & $172.0^{\circ}$ & $\mathrm{C} 2^{\prime}$-endo $\left({ }^{2} \mathrm{~T}_{3}\right)$ \\
\hline & B2(N3O4') & 43.4 & 43.5 & 42.5 & syn & $1.5^{\circ}$ & C3'-endo $\left({ }_{2}^{3} \mathrm{~T}\right)$ \\
\hline & B3(N3O4') & 46.4 & 47.4 & 43.6 & syn & 185.1 & C3'-exo $\left({ }_{3} \mathrm{~T}^{2}\right)$ \\
\hline & $\mathrm{B} 1\left(\mathrm{O} 4{ }^{\prime} \mathrm{O} 5{ }^{\prime}\right)$ & 52.2 & 51.9 & 50.5 & anti & $147.3^{\circ}$ & $\mathrm{C} 2^{\prime}$-endo $\left({ }^{2} \mathrm{~T}_{1}\right)$ \\
\hline & T2(N3O2'O3') & 52.2 & 53.6 & 50.8 & anti & $143.7^{\circ}$ & $\mathrm{C} 2$ '-endo $\left({ }_{1}^{2} \mathrm{~T}\right)$ \\
\hline & T3(N3O2'O3') & 52.2 & 53.6 & 50.9 & anti & $143.7^{\circ}$ & $\mathrm{C} 2$ '-endo $\left({ }_{1} \mathrm{~T}^{2}\right)$ \\
\hline & $\mathrm{B} 2\left(\mathrm{O} 4^{\prime} \mathrm{O} 5^{\prime}\right)$ & 55.7 & 56.2 & 52.1 & anti & $31.8^{\circ}$ & C3'-endo $\left({ }^{3} \mathrm{~T}_{4}\right)$ \\
\hline & $\mathrm{B} 1\left(\mathrm{~N}_{7} \mathrm{NH}_{2}\right)$ & 62.6 & 62.7 & 60.3 & anti & $168.0^{\circ}$ & $\mathrm{C} 2$ '-endo $\left({ }^{2} \mathrm{~T}_{3}\right)$ \\
\hline & $\mathrm{B} 2\left(\mathrm{~N} \mathrm{NH}_{2}\right)$ & 64.0 & 64.0 & 62.7 & anti & $11.0^{\circ}$ & C3'-endo $\left({ }^{3} \mathrm{~T}_{2}\right)$ \\
\hline & $\mathrm{B} 3\left(\mathrm{~N} \mathrm{NH} \mathrm{N}_{2}\right)$ & 62.5 & 61.2 & 63.2 & syn & $159.0^{\circ}$ & $\mathrm{C} 2$ '-endo $\left({ }^{2} \mathrm{~T}_{1}\right)$ \\
\hline & $\mathrm{B} 4\left(\mathrm{~N} \mathrm{NH} \mathrm{H}_{2}\right)$ & 63.2 & 62.0 & 63.5 & syn & $159.2^{\circ}$ & C2'-endo $\left({ }^{2} \mathrm{~T}_{1}\right)$ \\
\hline & $\mathrm{B} 5\left(\mathrm{~N} \mathrm{NH} \mathrm{H}_{2}\right)$ & 65.1 & 64.9 & 63.8 & anti & $13.1^{\circ}$ & C3'-endo $\left({ }^{3} \mathrm{~T}_{2}\right)$ \\
\hline & $\mathrm{B} 1\left(\mathrm{~N} 1 \mathrm{NH}_{2}\right)$ & 70.2 & 70.4 & 67.1 & anti & $170.6^{\circ}$ & $C 2$ '-endo $\left({ }^{2} \mathrm{~T}_{3}\right)$ \\
\hline & $\mathrm{B} 6\left(\mathrm{~N} \mathrm{NH}_{2}\right)$ & 73.0 & 73.5 & 68.9 & syn & 156.5 & C2'-endo $\left({ }^{2} \mathrm{~T}_{1}\right)$ \\
\hline & $\mathrm{B} 7\left(\mathrm{~N} \mathrm{NH} \mathrm{H}_{2}\right)$ & 74.5 & 75.2 & 69.1 & anti & $133.4^{\mathrm{o}}$ & C1'-exo $\left({ }_{1} \mathrm{~T}^{2}\right)$ \\
\hline & $\mathrm{B} 8\left(\mathrm{~N} \mathrm{NH} \mathrm{H}_{2}\right)$ & 73.0 & 73.1 & 69.3 & syn & $44.4^{\circ}$ & C4'-exo $\left({ }_{4} \mathrm{~T}^{3}\right)$ \\
\hline & $\mathrm{B} 9\left(\mathrm{~N}_{7} \mathrm{NH}_{2}\right)$ & 75.8 & 76.9 & 69.4 & anti & $136.5^{\circ}$ & C1'-exo $\left({ }_{1} \mathrm{~T}^{2}\right)$ \\
\hline & $\mathrm{B} 10\left(\mathrm{~N} \mathrm{NH}_{2}\right)$ & 69.0 & 67.8 & 69.8 & syn & $163.2^{\circ}$ & $\mathrm{C} 2$ '-endo $\left({ }^{2} \mathrm{E}\right)$ \\
\hline & $\mathrm{B} 2\left(\mathrm{~N} 1 \mathrm{NH}_{2}\right)$ & 74.2 & 74.5 & 71.1 & anti & $14.0^{\circ}$ & C3'-endo $\left({ }^{3} \mathrm{~T}_{2}\right)$ \\
\hline & $\mathrm{B} 11\left(\mathrm{~N} \mathrm{NH}_{2}\right)$ & 76.6 & 76.7 & 71.3 & anti & $32.1^{\circ}$ & C3'-endo $\left({ }^{3} \mathrm{~T}_{4}\right)$ \\
\hline & $\mathrm{B} 3\left(\mathrm{~N} 1 \mathrm{NH}_{2}\right)$ & 73.7 & 73.8 & 71.6 & anti & $173.0^{\circ}$ & $\mathrm{C} 2$ '-endo $\left({ }^{2} \mathrm{~T}_{3}\right)$ \\
\hline & $\mathrm{B} 12\left(\mathrm{~N} \mathrm{NH}_{2}\right)$ & 74.6 & 74.3 & 72.1 & anti & $151.7^{\circ}$ & $\mathrm{C} 2$ '-endo $\left({ }^{2} \mathrm{~T}_{1}\right)$ \\
\hline & $\mathrm{B} 4\left(\mathrm{~N} 1 \mathrm{NH}_{2}\right)$ & 75.0 & 75.2 & 72.4 & anti & $14.0^{\circ}$ & C3'-exo $\left({ }^{3} \mathrm{~T}_{2}\right)$ \\
\hline & $\mathrm{B} 5\left(\mathrm{~N} 1 \mathrm{NH}_{2}\right)$ & 80.2 & 81.1 & 73.0 & anti & $134.3^{\circ}$ & C1'-exo $\left({ }_{1} \mathrm{~T}^{2}\right)$ \\
\hline & $\mathrm{B} 6\left(\mathrm{~N}_{1} \mathrm{NH}_{2}\right)$ & 82.0 & 82.3 & 74.8 & anti & $46.4^{\circ}$ & $\mathrm{C} 44^{\prime}$-exo $\left({ }_{4} \mathrm{~T}^{3}\right)$ \\
\hline & $\mathrm{B} 7\left(\mathrm{~N} 1 \mathrm{NH}_{2}\right)$ & 77.0 & 77.2 & 74.9 & anti & $10.9^{\circ}$ & C3'-endo $\left({ }^{3} \mathrm{~T}_{2}\right)$ \\
\hline & B4(N3O4') & 77.3 & 78.1 & 74.9 & syn & $324.6^{\circ}$ & $\mathrm{C} 2^{\prime}$-exo $\left({ }_{2}^{1} \mathrm{~T}\right)$ \\
\hline & $\mathrm{B} 8\left(\mathrm{~N}_{1} \mathrm{NH}_{2}\right)$ & 82.6 & 83.3 & 77.0 & syn & $148.7^{\circ}$ & $\mathrm{C} 2$ '-endo $\left({ }^{2} \mathrm{~T}_{1}\right)$ \\
\hline & $\mathrm{B} 9\left(\mathrm{~N} 1 \mathrm{NH}_{2}\right)$ & 77.3 & 76.3 & 77.2 & syn & $161.6^{\circ}$ & $\mathrm{C} 2$ '-endo $\left({ }^{2} \mathrm{~T}_{1}\right)$ \\
\hline
\end{tabular}


Table S1. Relative Enthalpies and Free Energies of All Stable Low-Energy Conformers of $[\mathrm{dAdo}+\mathrm{Na}]^{+}$and $[\mathrm{Ado}+\mathrm{Na}]^{+}$at 0 and $298 \mathrm{~K}$ in $\mathrm{kJ} / \mathrm{mol}^{\alpha}$

\begin{tabular}{|c|c|c|c|c|c|c|c|}
\hline Species & Conformer & $\Delta \mathrm{H}_{0}$ & $\Delta \mathrm{H}_{298}$ & $\Delta \mathrm{G}_{298}$ & $\begin{array}{c}\text { Adenine } \\
\text { Orientation }\end{array}$ & $\mathrm{P}$ & Sugar Puckering \\
\hline \multirow[t]{19}{*}[\mathrm{Ado}+\mathrm{Na}]{$^{+}$} & $\mathrm{B} 10\left(\mathrm{~N} 1 \mathrm{NH}_{2}\right)$ & 82.3 & 82.7 & 77.3 & syn & $47.2^{\circ}$ & $\mathrm{C} 4$ '-exo $\left({ }_{4} \mathrm{~T}^{3}\right)$ \\
\hline & $\mathrm{B} 11\left(\mathrm{~N} 1 \mathrm{NH}_{2}\right)$ & 83.2 & 83.8 & 78.0 & syn & $149.5^{\circ}$ & C2'-endo $\left({ }^{2} \mathrm{~T}_{1}\right)$ \\
\hline & $\mathrm{B} 13\left(\mathrm{~N} 7 \mathrm{NH}_{2}\right)$ & 85.2 & 86.1 & 79.5 & syn & $161.0^{\circ}$ & $\mathrm{C} 2$ '-endo $\left({ }^{2} \mathrm{~T}_{1}\right)$ \\
\hline & $\mathrm{B} 14\left(\mathrm{~N} \mathrm{NH}_{2}\right)$ & 83.1 & 83.6 & 79.6 & anti & $185.8^{\circ}$ & C3'-exo $\left({ }_{3} T^{2}\right)$ \\
\hline & $\mathrm{B} 15\left(\mathrm{~N} \mathrm{NH}_{2}\right)$ & 82.4 & 82.2 & 79.7 & anti & $159.9^{\circ}$ & $\mathrm{C} 2$ '-endo $\left({ }^{2} \mathrm{~T}_{1}\right)$ \\
\hline & $\mathrm{B} 12\left(\mathrm{~N} 1 \mathrm{NH}_{2}\right)$ & 86.5 & 87.3 & 80.0 & anti & $136.1^{\circ}$ & C1'-exo $\left({ }_{1} \mathrm{~T}^{2}\right)$ \\
\hline & $\mathrm{B} 13\left(\mathrm{~N} 1 \mathrm{NH}_{2}\right)$ & 88.8 & 89.9 & 81.5 & anti & $133.5^{\circ}$ & C1'-exo $\left({ }_{1} \mathrm{~T}^{2}\right)$ \\
\hline & $\mathrm{B} 16\left(\mathrm{~N} \mathrm{NH}_{2}\right)$ & 86.7 & 86.8 & 82.3 & anti & $36.4^{\circ}$ & C3'-endo $\left({ }_{4}^{3} \mathrm{~T}\right)$ \\
\hline & $\mathrm{B} 13\left(\mathrm{~N} 1 \mathrm{NH}_{2}\right)$ & 86.9 & 87.1 & 82.6 & syn & $161.0^{\circ}$ & $\mathrm{C} 2$ '-endo $\left({ }^{2} \mathrm{~T}_{1}\right)$ \\
\hline & $\mathrm{B} 17\left(\mathrm{~N} 7 \mathrm{NH}_{2}\right)$ & 88.3 & 88.8 & 83.8 & syn & $53.1^{\circ}$ & C4'-exo $\left({ }_{4} \mathrm{~T}^{3}\right)$ \\
\hline & $\mathrm{B} 14\left(\mathrm{~N} 1 \mathrm{NH}_{2}\right)$ & 89.3 & 89.5 & 85.3 & anti & $162.8^{\circ}$ & $\mathrm{C} 2$ '-endo $\left({ }^{2} \mathrm{E}\right)$ \\
\hline & $\mathrm{B} 15\left(\mathrm{~N} 1 \mathrm{NH}_{2}\right)$ & 88.9 & 88.8 & 85.6 & anti & $163.4^{\circ}$ & $\mathrm{C} 2$ '-endo $\left({ }^{2} \mathrm{E}\right)$ \\
\hline & $\mathrm{B} 1$ (N7O5') & 84.6 & 83.5 & 86.1 & syn & $342.1^{\circ}$ & $\mathrm{C} 2^{\prime}-\operatorname{exo}\left({ }_{2} \mathrm{E}\right)$ \\
\hline & $\mathrm{B} 16\left(\mathrm{~N} 1 \mathrm{NH}_{2}\right)$ & 91.8 & 91.5 & 88.3 & anti & $150.5^{\circ}$ & C2'-endo $\left({ }^{2} \mathrm{~T}_{1}\right)$ \\
\hline & $\mathrm{B} 17\left(\mathrm{~N} 1 \mathrm{NH}_{2}\right)$ & 94.2 & 94.2 & 90.6 & anti & $157.3^{\circ}$ & C2'-endo $\left({ }^{2} \mathrm{~T}_{1}\right)$ \\
\hline & $\mathrm{B} 18\left(\mathrm{~N} 1 \mathrm{NH}_{2}\right)$ & 97.1 & 97.5 & 91.3 & anti & $36.9^{\circ}$ & C3'-endo $\left({ }_{4}^{3} \mathrm{~T}\right)$ \\
\hline & $\mathrm{B} 19\left(\mathrm{~N} 1 \mathrm{NH}_{2}\right)$ & 97.6 & 98.5 & 92.5 & syn & $159.4^{\circ}$ & C2'-endo $\left({ }^{2} \mathrm{~T}_{1}\right)$ \\
\hline & $\mathrm{M} 1(\mathrm{~N} 1)$ & 101.8 & 102.6 & 96.1 & anti & $152.2^{\circ}$ & $\mathrm{C} 2$ '-endo $\left({ }^{2} \mathrm{~T}_{1}\right)$ \\
\hline & $\mathrm{B} 20\left(\mathrm{~N} 1 \mathrm{NH}_{2}\right)$ & 99.3 & 99.6 & 96.3 & anti & $202.3^{\circ}$ & C3'-exo $\left({ }_{3} T^{4}\right)$ \\
\hline
\end{tabular}

${ }^{\alpha}$ Energetics based on single-point energy calculations performed at the B3LYP/6-311+G(2d,2p) level of theory, including ZPE and thermal corrections based on the B3LYP/6-311+G(d,p) optimized structures and vibrational frequencies 


\section{Figure Captions}

Figure S1. Designation of the conformational features of nucleosides including: nucleobase orientation and sugar puckering. The nucleobase orientation is designated as either anti or syn, depending upon the $\angle \mathrm{C} 4 \mathrm{~N}^{\prime} \mathrm{C}^{\prime}{ }^{\prime} \mathrm{O} 4^{\prime}$ dihedral angle, where conformations are designated as anti when the value of this angle lies between $90^{\circ}$ and $270^{\circ}$, and as $s y n$ when the value of this angle lies between $-90^{\circ}$ and $90^{\circ}$. Two different designations for the sugar puckering are shown that are both based on the pseudorotation phase angle, $\mathrm{P}$, as defined by Saenger and computed using eq (S1) (reference 3 of main text),

$$
\tan P=\frac{\left(v_{4}+v_{1}\right)-\left(v_{3}+v_{0}\right)}{2 \times v_{2} \times\left(\sin 36^{\circ}+\sin 72^{\circ}\right)}
$$

where the angles, $v_{0}, v_{1}, v_{2}, v_{3}$ and $v_{4}$, represent the $\angle \mathrm{C}^{\prime} \mathrm{O} 4^{\prime} \mathrm{C} 1^{\prime} \mathrm{C} 2^{\prime}, \angle \mathrm{O} 4^{\prime} \mathrm{C} 1^{\prime} \mathrm{C} 2^{\prime} \mathrm{C} 3^{\prime}, \angle \mathrm{C} 1^{\prime} \mathrm{C} 2^{\prime} \mathrm{C} 3^{\prime} \mathrm{C} 4^{\prime}, \angle \mathrm{C} 2^{\prime} \mathrm{C} 3^{\prime} \mathrm{C} 4^{\prime} \mathrm{O} 4^{\prime}$ and $\angle \mathrm{C}^{\prime} \mathrm{C}^{\prime} \mathrm{O} 4^{\prime} \mathrm{C} 1^{\prime}$ dihedral angles, respectively. In the upper section of the diagram, $v_{2}>0$, whereas in the lower section of the diagram, $v_{2}<0$. The combination of $\mathrm{P}$ and $v_{2}$ can be used to identify the sugar puckering. Classical designations based on endo and exo configurations that designate the single atom that deviates most significantly from the plane of the sugar moiety are shown. However, the sugar can be puckered in an envelope (E) form where four of the ring atoms essentially lie in a plane as assumed based on the classical nomenclature, or in a twist (T) form with two adjacent atoms displaced on opposite sides of the plane defined by other three atoms. For the designations, superscripts are used to indicate atoms that lie above the plane, endo (on the same side as the C5' atom), whereas subscripts indicate atoms that lie below the plane, or exo. Atom numbering before the T or E designation represent the major puckering, whereas atoms that follow designate the minor puckering. The classical designation is based on variation in $\mathrm{P}$ that are multiples of $36^{\circ}$, whereas the $\mathrm{E}$ and $\mathrm{T}$ forms alternate every $18^{\circ}$. The mirror sugar puckering of the starting position is found after rotation by $180^{\circ}$.

Figure S2. Stable low-energy conformers of $[\mathrm{dAdo}+\mathrm{Na}]^{+}$determined at the B3LYP/6-311+G(2d,2p)//B3LYP/6-311+G(d,p) level of theory that are within $100 \mathrm{~kJ} / \mathrm{mol}$ of the ground-state conformer. The $\mathrm{Na}^{+}$binding modes, orientations of adenine, sugar configurations, and the relative Gibbs free energies at $298 \mathrm{~K}$ are also listed for each structure.

Figure S3. Stable low-energy conformers of $[\mathrm{Ado}+\mathrm{Na}]^{+}$determined at the $\mathrm{B} 3 \mathrm{LYP} / 6-311+\mathrm{G}(2 \mathrm{~d}, 2 \mathrm{p}) / / \mathrm{B} 3 \mathrm{LYP} / 6-311+\mathrm{G}(\mathrm{d}, \mathrm{p})$ level of theory that are within $100 \mathrm{~kJ} / \mathrm{mol}$ of the ground-state conformer. The $\mathrm{Na}^{+}$binding modes, orientations of adenine, sugar configurations, and the relative Gibbs free energies at $298 \mathrm{~K}$ are also listed for each structure.

Figure S4. Comparison of the experimental IRMPD action spectrum of [dAdo+Na] ${ }^{+}$with the B3LYP/6-311+G(d,p) optimized structures and calculated linear IR spectra for several representative stable low-energy conformers of $[\mathrm{dAdo}+\mathrm{Na}]^{+}$. The B3LYP/6-311+G(2d,2p) relative Gibbs free energies at $298 \mathrm{~K}$ are also shown.

Figure S5. Comparison of the experimental IRMPD action spectrum of [Ado+Na] ${ }^{+}$with the B3LYP/6-311+G(d,p) optimized structures and calculated linear IR spectra for several representative stable low-energy conformers of $[\text { Ado }+\mathrm{Na}]^{+}$. The B3LYP/6-311+G(2d,2p) relative Gibbs free energies at $298 \mathrm{~K}$ are also shown. 
Figure S1.

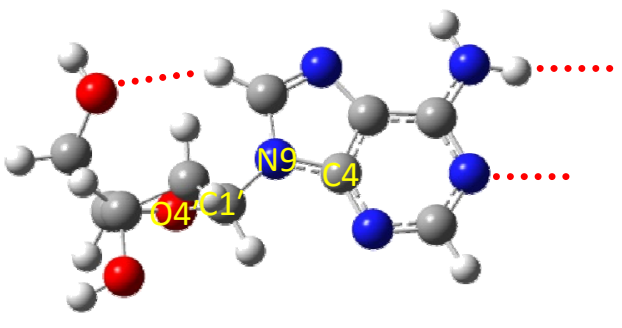

anti orientation

$\angle \mathrm{C} 4 \mathrm{~N} 9 \mathrm{C} 1^{\prime} \mathrm{O} 4^{\prime}=90^{\circ}$ to $270^{\circ}$

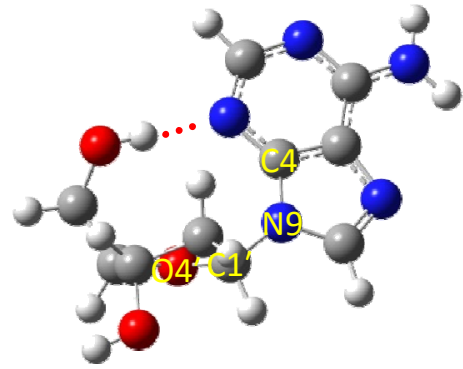

syn orientation $\angle \mathrm{C} 4 \mathrm{~N} 9 \mathrm{C} 1^{\prime} \mathrm{O} 4^{\prime}=-90^{\circ}$ to $90^{\circ}$

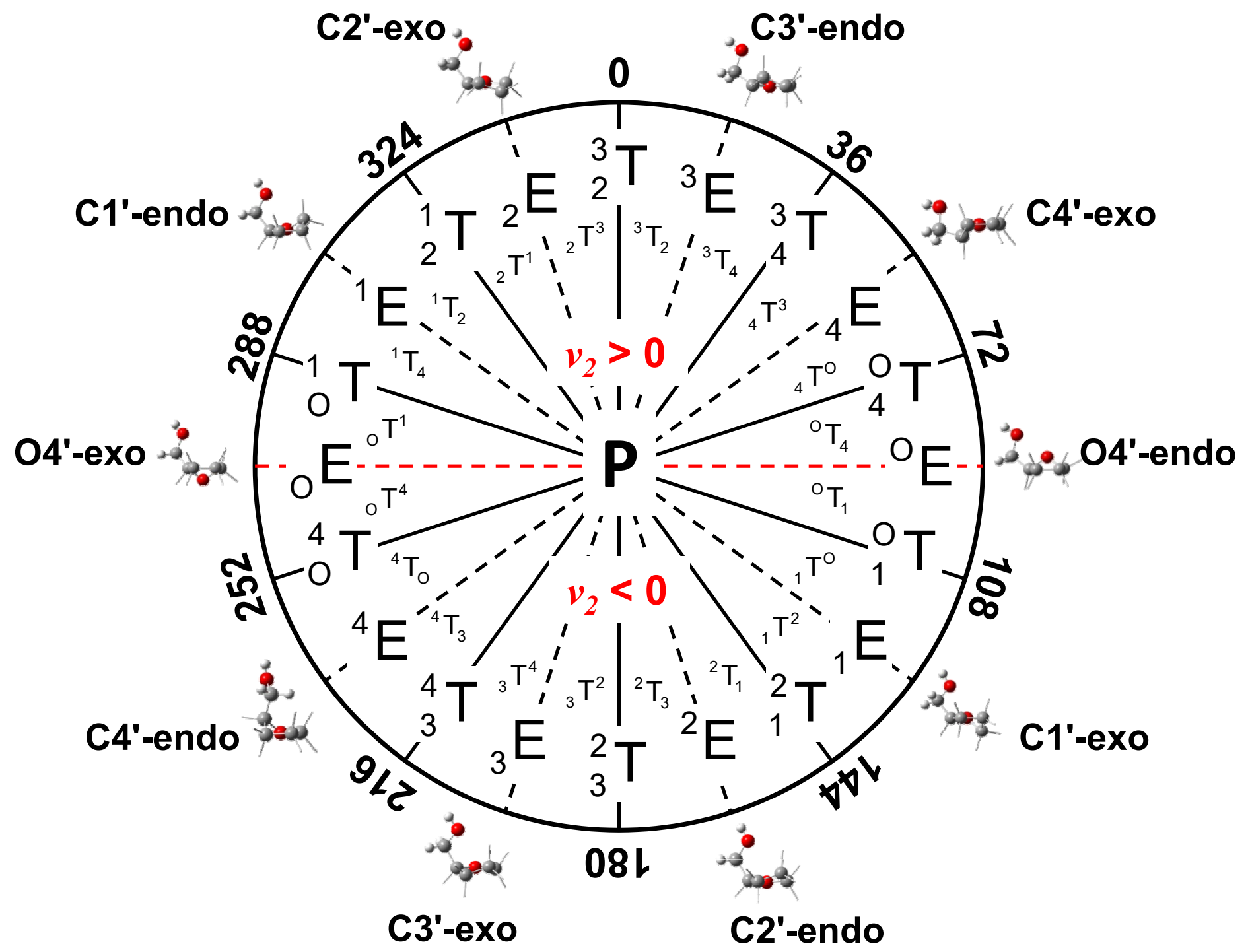


Figure S2.

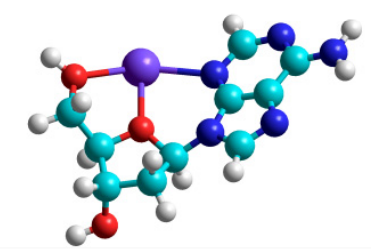

T1(N3O4'O5')

syn, C1'-exo $\left({ }_{1}{ }^{2} \mathrm{~T}\right)$

$0.0 \mathrm{~kJ} / \mathrm{mol}$

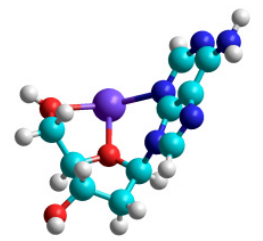

T5(N3O4'O5')

syn, C2'-exo $\left({ }_{2} \mathrm{~T}^{3}\right)$

$3.5 \mathrm{~kJ} / \mathrm{mol}$

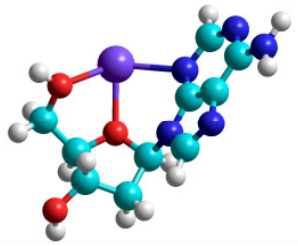

T9(N3O4'O5')

syn, C3'-endo $\left({ }^{3} \mathrm{~T}_{2}\right)$

$29.5 \mathrm{~kJ} / \mathrm{mol}$

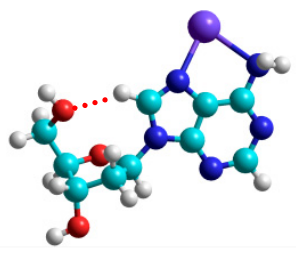

$\mathrm{B} 1\left(\mathrm{~N} \mathrm{NH}_{2}\right)$

anti, $\mathrm{C} 2$ '-endo $\left({ }^{2} \mathrm{~T}_{3}\right)$

$65.0 \mathrm{~kJ} / \mathrm{mol}$

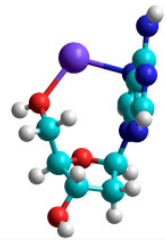

B1(N7O5')

anti, $\mathrm{C} 1$ '-endo $\left({ }^{1} \mathrm{~T}_{4}\right)$

$72.6 \mathrm{~kJ} / \mathrm{mol}$

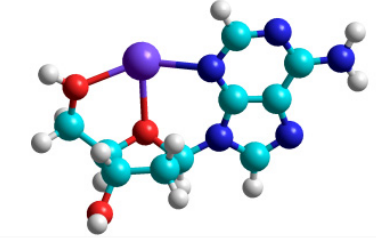

T2(N3O4'O5')

syn, $\mathrm{O}^{\prime}$-endo $\left({ }^{\circ} \mathrm{T}_{4}\right)$

$1.4 \mathrm{~kJ} / \mathrm{mol}$

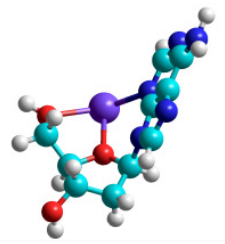

T6(N3O4'O5')

syn, C2'-exo $\left({ }_{2} \mathrm{~T}^{3}\right)$

$9.9 \mathrm{~kJ} / \mathrm{mol}$

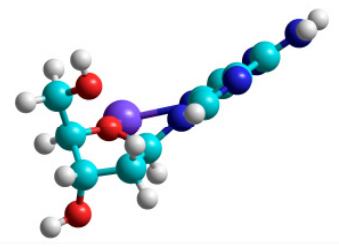

B1(N3O4')

syn, C3'-exo $\left({ }_{3} \mathrm{~T}^{2}\right)$

$33.7 \mathrm{~kJ} / \mathrm{mol}$

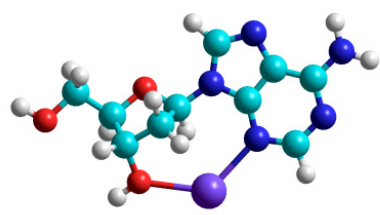

B1(N3O3')

anti, $\mathrm{C} 1$ '-exo $\left({ }_{1} \mathrm{~T}^{2}\right)$

$66.4 \mathrm{~kJ} / \mathrm{mol}$

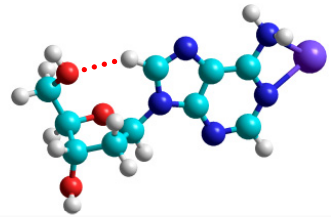

$\mathrm{B} 1\left(\mathrm{~N} 1 \mathrm{NH}_{2}\right)$

anti, C2'-endo $\left({ }^{2} \mathrm{~T}_{3}\right)$

$73.1 \mathrm{~kJ} / \mathrm{mol}$

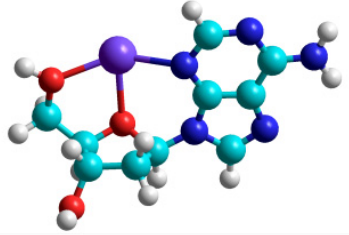

T3(N3O4'O5')

syn, $\mathrm{O}^{\prime}$ '-endo $\left({ }^{\circ} \mathrm{T}_{4}\right)$

$1.5 \mathrm{~kJ} / \mathrm{mol}$

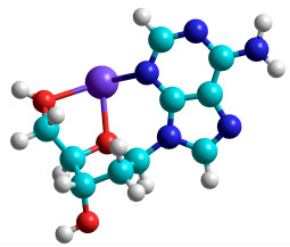

T7(N3O4'O5')

syn, , C2'-endo $\left({ }^{2} \mathrm{~T}_{1}\right)$ $10.3 \mathrm{~kJ} / \mathrm{mol}$

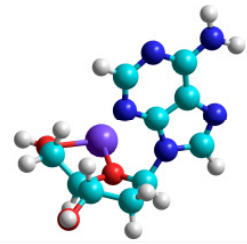

$\mathrm{B} 1\left(\mathrm{O} 4 \mathrm{O}^{\prime}\right)$

syn, C3'-endo $\left({ }^{3} \mathrm{~T}_{2}\right)$

$41.4 \mathrm{~kJ} / \mathrm{mol}$

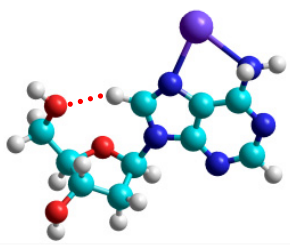

$\mathrm{B} 2\left(\mathrm{~N}_{7} \mathrm{NH}_{2}\right)$

anti, $\mathrm{C}^{3}$ '-endo $\left({ }^{3} \mathrm{~T}_{2}\right)$

$69.3 \mathrm{~kJ} / \mathrm{mol}$

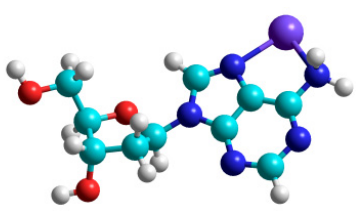

$\mathrm{B} 4\left(\mathrm{~N}_{7} \mathrm{NH}_{2}\right)$

anti, C2'-endo $\left({ }^{2} \mathrm{~T}_{1}\right)$

$74.1 \mathrm{~kJ} / \mathrm{mol}$

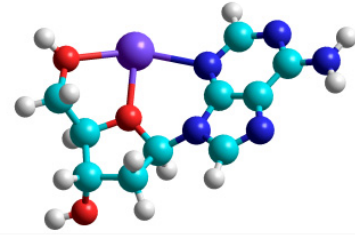

T4(N3O4'O5')

syn, C2'-endo $\left({ }^{2} \mathrm{~T}_{1}\right)$

$2.2 \mathrm{~kJ} / \mathrm{mol}$

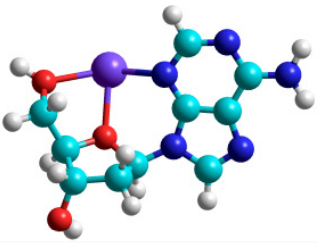

T8(N3O4'O5')

syn, C1'-exo $\left({ }_{1} \mathrm{~T}^{2}\right)$

$20.4 \mathrm{~kJ} / \mathrm{mol}$

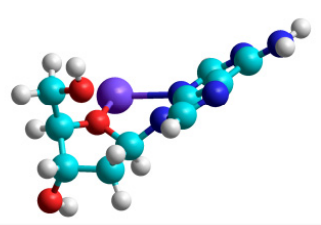

$\mathrm{B} 2\left(\mathrm{~N} 3 \mathrm{O} 4{ }^{\prime}\right)$

syn, C3'-exo $\left({ }_{3} \mathrm{~T}^{4}\right)$

$48.3 \mathrm{~kJ} / \mathrm{mol}$

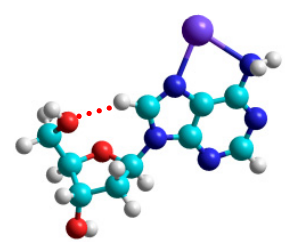

$\mathrm{B} 3\left(\mathrm{~N} \mathrm{NH}_{2}\right)$

anti, $\mathrm{C} 2$ '-endo $\left({ }^{2} \mathrm{~T}_{3}\right)$

$72.5 \mathrm{~kJ} / \mathrm{mol}$

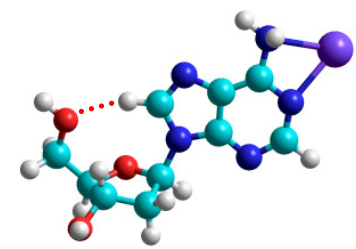

$\mathrm{B} 2\left(\mathrm{~N} 1 \mathrm{NH}_{2}\right)$

anti, $\mathrm{C}^{\prime}$-endo $\left({ }^{3} \mathrm{~T}_{2}\right)$

$74.4 \mathrm{~kJ} / \mathrm{mol}$ 
Figure S2.

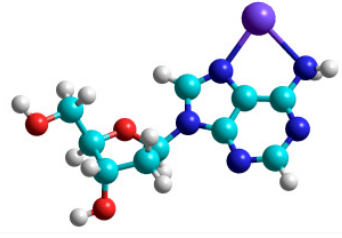

$\mathrm{B} 5\left(\mathrm{~N} \mathrm{NH}_{2}\right)$

anti, C2'-endo $\left({ }^{2} \mathrm{~T}_{1}\right)$

$74.8 \mathrm{~kJ} / \mathrm{mol}$

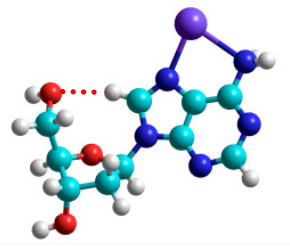

$\mathrm{B} 9\left(\mathrm{~N} 7 \mathrm{NH}_{2}\right)$

anti, C3'-exo $\left({ }_{3} \mathrm{~T}^{4}\right)$

$76.0 \mathrm{~kJ} / \mathrm{mol}$

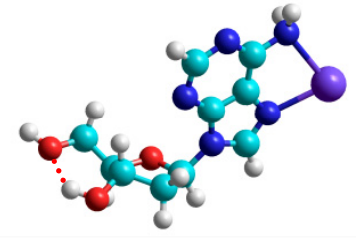

$\mathrm{B} 12\left(\mathrm{~N} \mathrm{NH}_{2}\right)$

syn, C4'-exo $\left({ }_{4} \mathrm{~T}^{3}\right)$

$77.9 \mathrm{~kJ} / \mathrm{mol}$

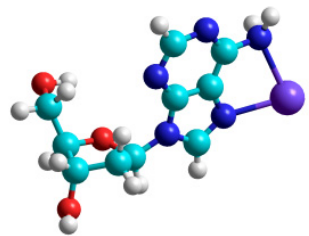

$\mathrm{B} 13\left(\mathrm{~N} 7 \mathrm{NH}_{2}\right)$

syn, C2'-endo ( $\left.{ }^{2} \mathrm{E}\right)$

$82.4 \mathrm{~kJ} / \mathrm{mol}$

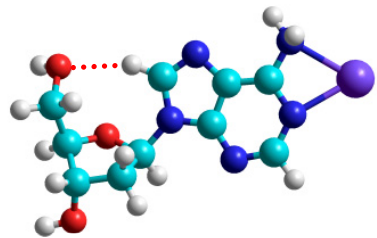

$\mathrm{B} 6\left(\mathrm{~N} 1 \mathrm{NH}_{2}\right)$

anti, C3'-exo $\left({ }_{3} \mathrm{~T}^{4}\right)$

$87.7 \mathrm{~kJ} / \mathrm{mol}$

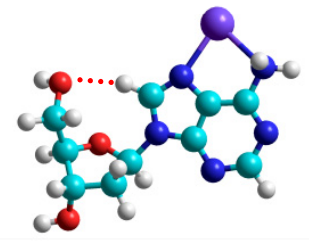

$\mathrm{B} 6\left(\mathrm{~N} 7 \mathrm{NH}_{2}\right)$

anti, C3'-exo $\left({ }_{3} \mathrm{~T}^{4}\right)$

$75.5 \mathrm{~kJ} / \mathrm{mol}$

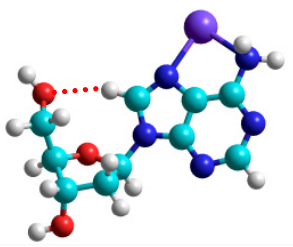

$\mathrm{B} 10\left(\mathrm{~N} \mathrm{NH}_{2}\right)$

anti, C3'-exo $\left({ }_{3} \mathrm{~T}^{4}\right)$

$76.8 \mathrm{~kJ} / \mathrm{mol}$

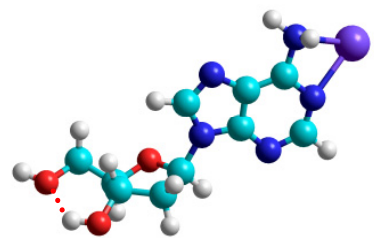

$\mathrm{B} 4\left(\mathrm{~N} 1 \mathrm{NH}_{2}\right)$

anti, C4'-exo $\left({ }_{4} \mathrm{~T}^{3}\right)$

$79.0 \mathrm{~kJ} / \mathrm{mol}$

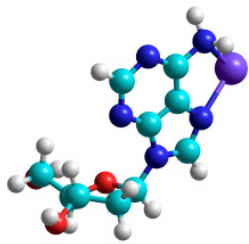

$\mathrm{B} 14\left(\mathrm{~N} \mathrm{NH}_{2}\right)$

syn, C4'-exo $\left({ }_{4} \mathrm{~T}^{3}\right)$

$83.1 \mathrm{~kJ} / \mathrm{mol}$

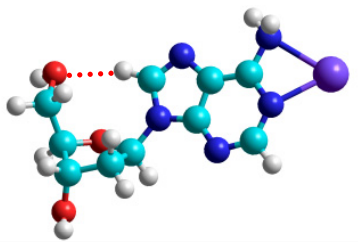

$\mathrm{B} 7\left(\mathrm{~N}_{1} \mathrm{NH}_{2}\right)$

anti, C3'-exo $\left({ }_{3} \mathrm{E}\right)$

$88.3 \mathrm{~kJ} / \mathrm{mol}$

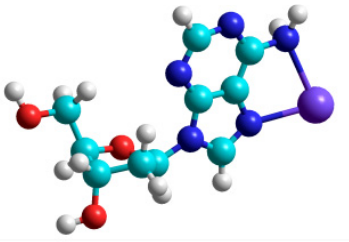

$\mathrm{B} 7\left(\mathrm{~N} 7 \mathrm{NH}_{2}\right)$

syn, C2'-endo $\left({ }^{2} \mathrm{~T}_{3}\right)$

$75.7 \mathrm{~kJ} / \mathrm{mol}$

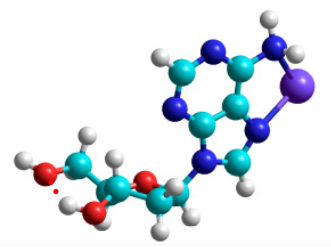

$\mathrm{B} 11\left(\mathrm{~N} 7 \mathrm{NH}_{2}\right)$

syn, C4'-exo $\left({ }_{4} \mathrm{~T}^{3}\right)$

$77.4 \mathrm{~kJ} / \mathrm{mol}$

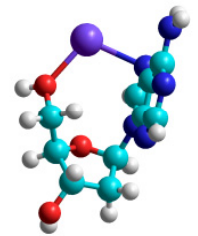

B1(N305')

anti, C1'-endo (o $\left.{ }^{1} \mathrm{~T}\right)$

$79.5 \mathrm{~kJ} / \mathrm{mol}$

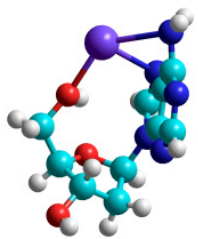

T1(N7NH $\left.{ }_{2} \mathrm{O5}^{\prime}\right)$

anti, C2'-exo $\left({ }_{2} \mathrm{~T}^{1}\right)$

$85.2 \mathrm{~kJ} / \mathrm{mol}$

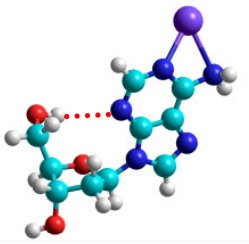

$\mathrm{B} 8\left(\mathrm{~N} 1 \mathrm{NH}_{2}\right)$

syn, C2'-endo $\left({ }^{2} \mathrm{~T}_{1}\right)$

$88.4 \mathrm{~kJ} / \mathrm{mol}$

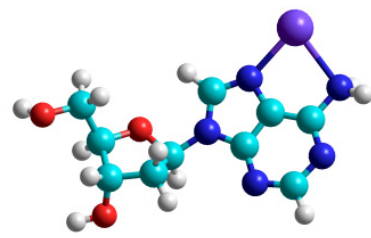

$\mathrm{B} 8\left(\mathrm{~N} \mathrm{NH}_{2}\right)$

anti, C2'-endo $\left({ }^{2} \mathrm{~T}_{1}\right)$

$75.8 \mathrm{~kJ} / \mathrm{mol}$

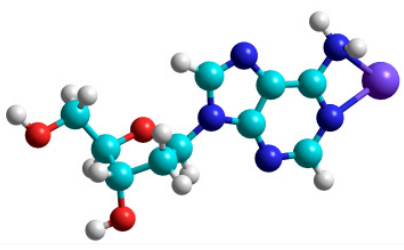

$\mathrm{B} 3\left(\mathrm{~N} 1 \mathrm{NH}_{2}\right)$

anti, C2'-endo $\left({ }^{2} \mathrm{~T}_{1}\right)$

$77.6 \mathrm{~kJ} / \mathrm{mol}$

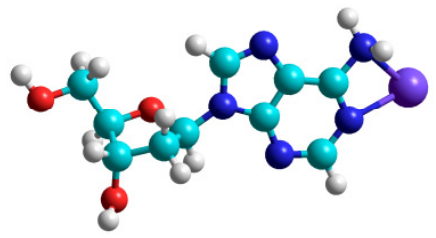

$\mathrm{B} 5\left(\mathrm{~N} 1 \mathrm{NH}_{2}\right)$

anti, C2'-endo $\left({ }^{2} \mathrm{~T}_{1}\right)$

$81.2 \mathrm{~kJ} / \mathrm{mol}$

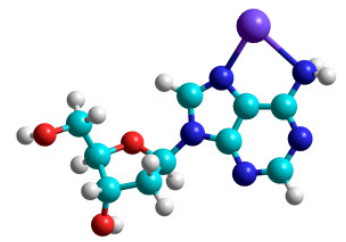

$\mathrm{B} 15\left(\mathrm{~N} 7 \mathrm{NH}_{2}\right)$

anti, C2'-endo ( $\left.{ }^{2} \mathrm{~T}_{3}\right)$

$87.3 \mathrm{~kJ} / \mathrm{mol}$

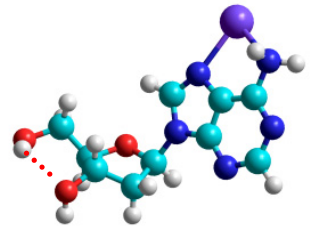

$\mathrm{B} 16\left(\mathrm{~N} \mathrm{NH}_{2}\right)$

anti, C3'-endo $\left({ }^{3} \mathrm{~T}_{4}\right)$

$88.6 \mathrm{~kJ} / \mathrm{mol}$ 


\section{S10 \\ Figure S2.}

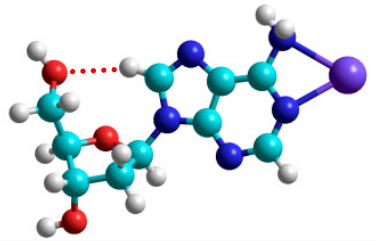

$\mathrm{B} 9\left(\mathrm{~N} 1 \mathrm{NH}_{2}\right)$

anti, C3'-exo $\left({ }_{3} \mathrm{~T}^{4}\right)$ $89.0 \mathrm{~kJ} / \mathrm{mol}$

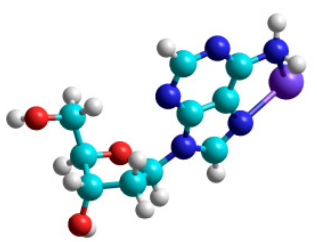

B17 $\left(\mathrm{N} \mathrm{NH}_{2}\right)$

syn, $\mathrm{C}^{\prime}-\mathrm{exo}\left({ }_{3} \mathrm{~T}^{2}\right)$ $91.6 \mathrm{~kJ} / \mathrm{mol}$

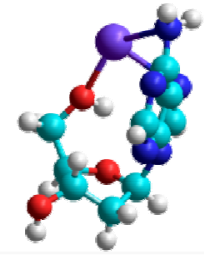

$\mathrm{T} 2\left(\mathrm{~N} \mathrm{NH}_{2} \mathrm{O}^{\prime}\right)$

anti, C2'-exo $\left({ }_{2} \mathrm{~T}^{1}\right)$

$90.3 \mathrm{~kJ} / \mathrm{mol}$

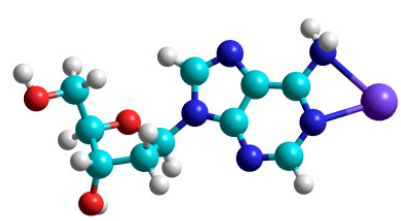

$\mathrm{B} 11\left(\mathrm{~N} 1 \mathrm{NH}_{2}\right)$

anti, C2'-endo $\left({ }^{2} \mathrm{~T}_{3}\right)$

$93.0 \mathrm{~kJ} / \mathrm{mol}$

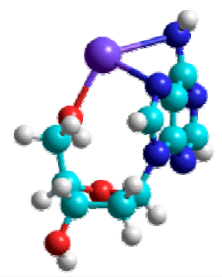

T3 $\left(\mathrm{N}^{\prime} \mathrm{NH}_{2} \mathrm{O}^{\prime}\right)$

syn, C4'-endo $\left({ }^{4} \mathrm{~T}_{\mathrm{O}}\right)$ $90.9 \mathrm{~kJ} / \mathrm{mol}$

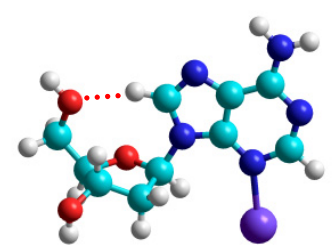

$\mathrm{M} 1$ (N3)

anti, C3'-endo $\left({ }^{3} \mathrm{~T}_{2}\right)$ $95.4 \mathrm{~kJ} / \mathrm{mol}$

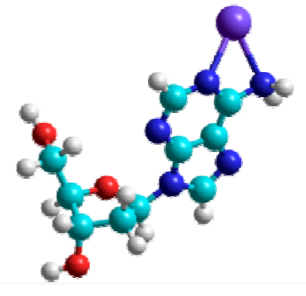

$\mathrm{B} 10\left(\mathrm{~N} 1 \mathrm{NH}_{2}\right)$

syn, C2'-endo $\left({ }^{2} \mathrm{~T}_{3}\right)$

$91.2 \mathrm{~kJ} / \mathrm{mol}$

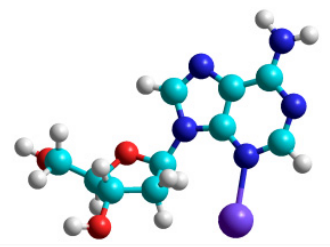

M2(N3)

anti, C4'-exo $\left({ }_{4} \mathrm{E}\right)$ $99.9 \mathrm{~kJ} / \mathrm{mol}$

$[\mathrm{dAdo}+\mathrm{Na}]^{+}$ 
Figure S3.

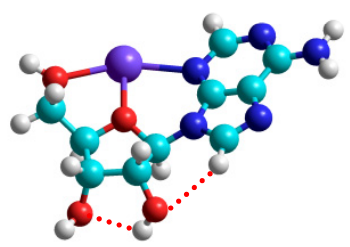

T1(N3O4'O5')

syn, $\mathrm{C} 1$ '-exo $\left({ }_{1} \mathrm{~T}^{\mathrm{O}}\right)$

$0.0 \mathrm{~kJ} / \mathrm{mol}$

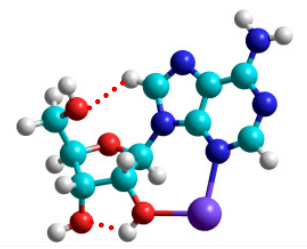

B2(N3O2')

anti, C3'-exo $\left({ }_{3} \mathrm{~T}^{4}\right)$

$21.2 \mathrm{~kJ} / \mathrm{mol}$

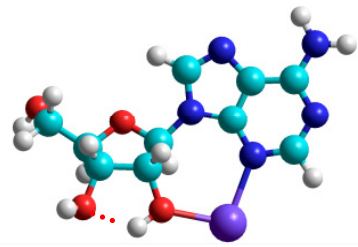

B6(N3O2')

anti, $\mathrm{O}^{\prime}$ '-endo $\left(\mathrm{O}_{4}\right)$

$33.2 \mathrm{~kJ} / \mathrm{mol}$

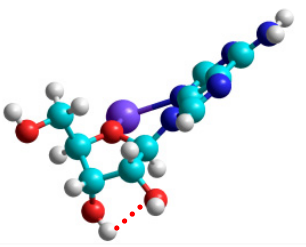

B3(N3O4')

syn, C3'-exo $\left({ }_{3} \mathrm{~T}^{2}\right)$

$43.6 \mathrm{~kJ} / \mathrm{mol}$

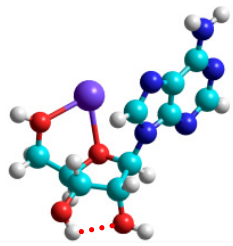

$\mathrm{B} 2\left(\mathrm{O} 4 \mathrm{O}^{\prime}\right)$

anti, C3'-endo $\left({ }^{3} \mathrm{~T}_{4}\right)$

$52.1 \mathrm{~kJ} / \mathrm{mol}$

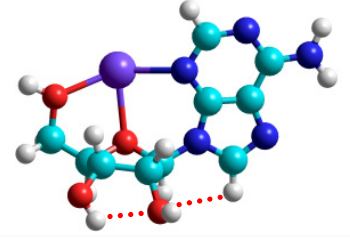

T2(N3O4'O5')

syn, $\mathrm{O}^{\prime}$ '-endo $\left({ }^{\circ} \mathrm{T}_{4}\right)$

$1.0 \mathrm{~kJ} / \mathrm{mol}$

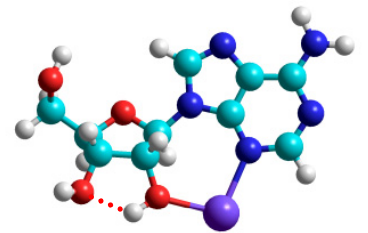

B3(N3O2')

anti, $\mathrm{O}^{\prime}$-endo $\left({ }^{\circ} \mathrm{T}_{4}\right)$

$21.3 \mathrm{~kJ} / \mathrm{mol}$

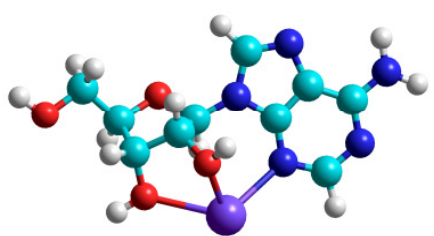

T1(N3O2'O3')

anti, $\mathrm{C} 1$ '-exo $\left({ }_{1} \mathrm{~T}^{2}\right)$

$36.0 \mathrm{~kJ} / \mathrm{mol}$

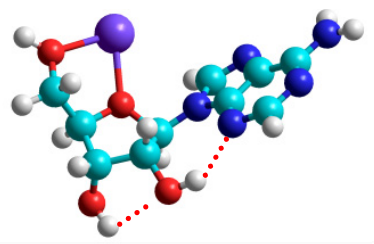

B1(O4'O5')

anti, C2'-endo $\left({ }^{2} \mathrm{~T}_{1}\right)$

$50.5 \mathrm{~kJ} / \mathrm{mol}$

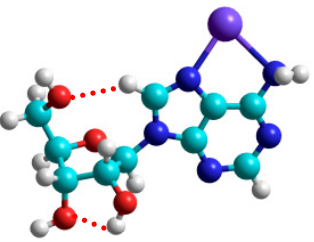

$\mathrm{B} 1\left(\mathrm{~N}_{\mathrm{N}} \mathrm{NH}_{2}\right)$

anti, C2'-endo $\left({ }^{2} \mathrm{~T}_{3}\right)$

$60.3 \mathrm{~kJ} / \mathrm{mol}$

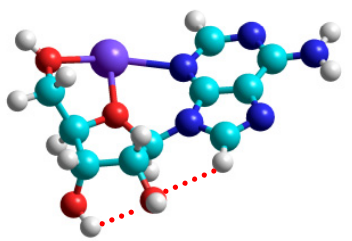

T3(N3O4'O5')

syn, $\mathrm{C} 1$ '-exo $\left({ }_{1} \mathrm{~T}^{2}\right)$

$4.3 \mathrm{~kJ} / \mathrm{mol}$

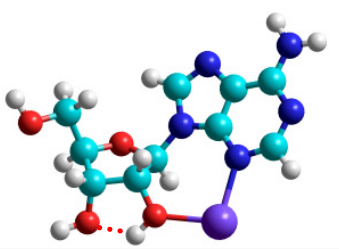

B4(N3O2')

anti, C3'-exo $\left({ }_{3} \mathrm{E}\right)$

$27.9 \mathrm{~kJ} / \mathrm{mol}$

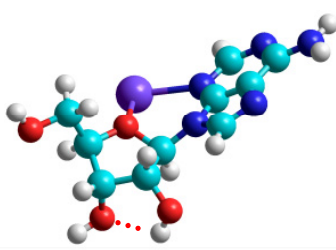

B1(N3O4')

syn, $\mathrm{C} 2$ '-endo $\left({ }^{2} \mathrm{~T}_{3}\right)$

$39.5 \mathrm{~kJ} / \mathrm{mol}$

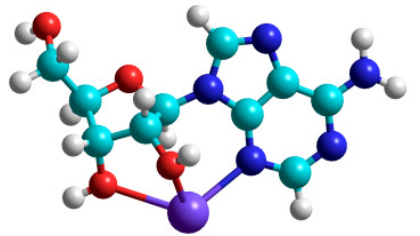

T2(N3O2'O3')

anti, C2'-endo $\left({ }_{1}^{2} \mathrm{~T}\right)$

$50.8 \mathrm{~kJ} / \mathrm{mol}$

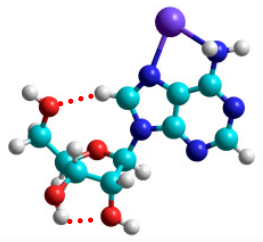

$\mathrm{B} 2\left(\mathrm{~N}_{7} \mathrm{NH}_{2}\right)$

anti, $\mathrm{C} 3$ '-endo $\left({ }^{3} \mathrm{~T}_{2}\right)$

$62.7 \mathrm{~kJ} / \mathrm{mol}$

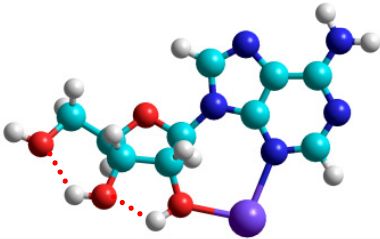

$\mathrm{B} 1\left(\mathrm{~N} 3 \mathrm{O} 2{ }^{\prime}\right)$

anti, $\mathrm{C}^{\prime}$-exo $\left({ }_{4} \mathrm{~T}^{\mathrm{O}}\right)$

$19.7 \mathrm{~kJ} / \mathrm{mol}$

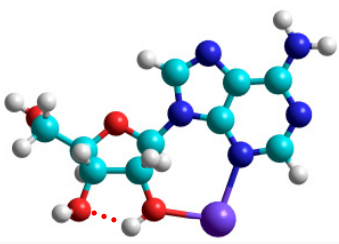

B5(N3O2')

anti, $\mathrm{O}^{\prime}$ '-endo $\left({ }^{\circ} \mathrm{T}_{4}\right)$ $30.2 \mathrm{~kJ} / \mathrm{mol}$

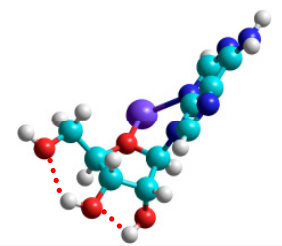

$\mathrm{B} 2(\mathrm{~N} 3 \mathrm{O} 4$ ')

syn, C3'-endo $\left({ }_{2}{ }^{3} \mathrm{~T}\right)$

$42.5 \mathrm{~kJ} / \mathrm{mol}$

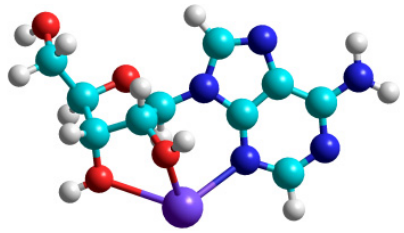

T3(N3O2'O3')

anti, $\mathrm{C} 2$ '-endo $\left({ }_{1} \mathrm{~T}^{2}\right)$

$50.9 \mathrm{~kJ} / \mathrm{mol}$

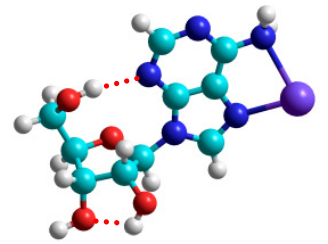

$\mathrm{B} 3\left(\mathrm{~N}_{7} \mathrm{NH}_{2}\right)$

syn, $\mathrm{C} 2$ '-endo $\left({ }^{2} \mathrm{~T}_{1}\right)$

$63.2 \mathrm{~kJ} / \mathrm{mol}$ 
Figure S3.

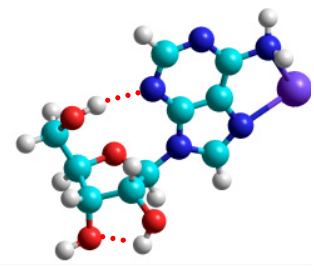

$\mathrm{B} 4\left(\mathrm{~N} \mathrm{NH}_{2}\right)$

syn, C2'-endo $\left({ }^{2} \mathrm{~T}_{1}\right)$

$63.5 \mathrm{~kJ} / \mathrm{mol}$

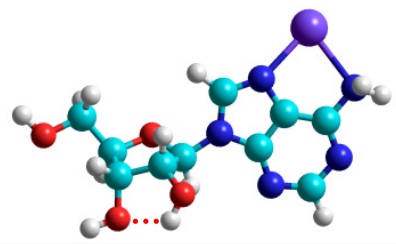

$\mathrm{B} 7\left(\mathrm{~N} \mathrm{NH}_{2}\right)$

anti, $\mathrm{C} 1$ '-exo $\left({ }_{1} \mathrm{~T}^{2}\right)$

$69.1 \mathrm{~kJ} / \mathrm{mol}$

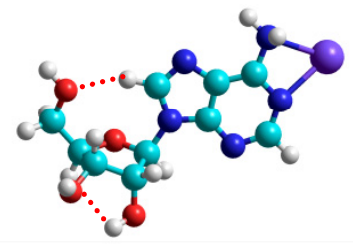

$\mathrm{B} 2\left(\mathrm{~N} 1 \mathrm{NH}_{2}\right)$

anti, C3'-endo $\left({ }^{3} \mathrm{~T}_{2}\right)$

$71.1 \mathrm{~kJ} / \mathrm{mol}$

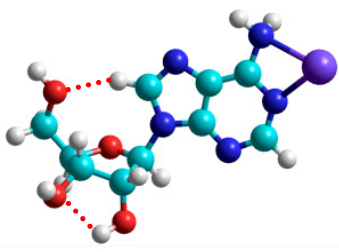

$\mathrm{B} 4\left(\mathrm{~N} 1 \mathrm{NH}_{2}\right)$

anti, C3'-exo $\left({ }^{3} \mathrm{~T}_{2}\right)$

$72.4 \mathrm{~kJ} / \mathrm{mol}$

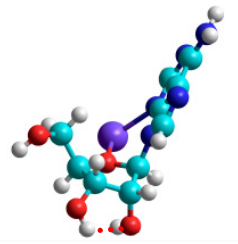

$\mathrm{B} 4\left(\mathrm{~N} 3 \mathrm{O} 4{ }^{\prime}\right)$

syn, C2'-exo $\left({ }_{2}^{1} \mathrm{~T}\right)$

$74.9 \mathrm{~kJ} / \mathrm{mol}$

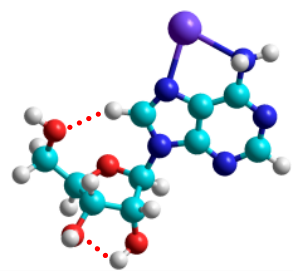

$\mathrm{B} 5\left(\mathrm{~N} 7 \mathrm{NH}_{2}\right)$

anti, $\mathrm{C}^{\prime}$-endo $\left({ }^{3} \mathrm{~T}_{2}\right)$

$63.8 \mathrm{~kJ} / \mathrm{mol}$

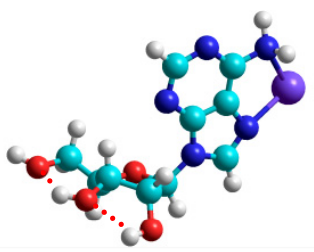

$\mathrm{B} 8\left(\mathrm{~N} \mathrm{NH}_{2}\right)$

syn, $\mathrm{C}^{\prime}$-exo $\left({ }_{4} \mathrm{~T}^{3}\right)$

$69.3 \mathrm{~kJ} / \mathrm{mol}$

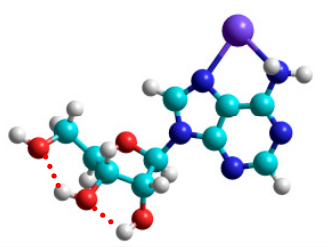

$\mathrm{B} 11\left(\mathrm{~N} 7 \mathrm{NH}_{2}\right)$

anti, $\mathrm{C}^{\prime}$-endo $\left({ }^{3} \mathrm{~T}_{4}\right)$

$71.3 \mathrm{~kJ} / \mathrm{mol}$

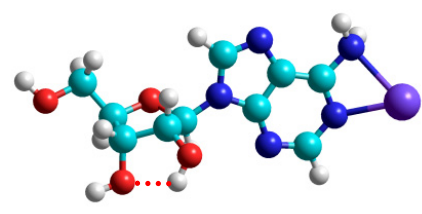

$\mathrm{B} 5\left(\mathrm{~N} 1 \mathrm{NH}_{2}\right)$

anti, $\mathrm{C} 1$ '-exo $\left({ }_{1} \mathrm{~T}^{2}\right)$

$73.0 \mathrm{~kJ} / \mathrm{mol}$

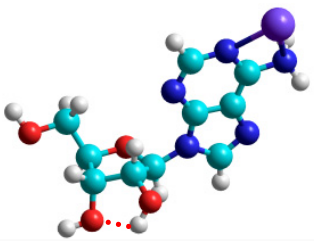

$\mathrm{B} 8\left(\mathrm{~N}_{1} \mathrm{NH}_{2}\right)$

syn, C2'-endo $\left({ }^{2} \mathrm{~T}_{1}\right)$

$77.0 \mathrm{~kJ} / \mathrm{mol}$

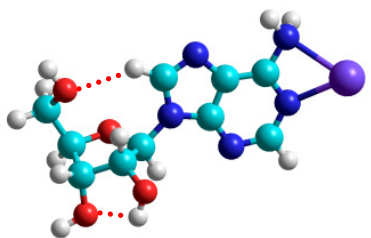

$\mathrm{B} 1\left(\mathrm{~N} 1 \mathrm{NH}_{2}\right)$

anti, $\mathrm{C} 2$ '-endo $\left({ }^{2} \mathrm{~T}_{3}\right)$

$67.1 \mathrm{~kJ} / \mathrm{mol}$

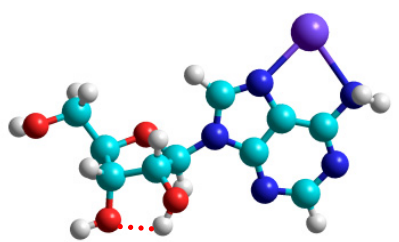

$\mathrm{B} 9\left(\mathrm{~N}_{\mathrm{NH}} \mathrm{NH}_{2}\right.$

anti, C1'-exo $\left({ }_{1} \mathrm{~T}^{2}\right)$

$69.4 \mathrm{~kJ} / \mathrm{mol}$

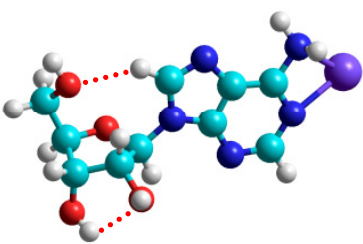

$\mathrm{B} 3\left(\mathrm{~N} 1 \mathrm{NH}_{2}\right)$

anti, C2'-endo $\left({ }^{2} \mathrm{~T}_{3}\right)$

$71.6 \mathrm{~kJ} / \mathrm{mol}$

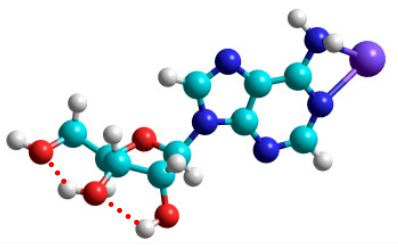

$\mathrm{B} 6\left(\mathrm{~N}_{1} \mathrm{NH}_{2}\right)$

anti, C4'-exo $\left({ }_{4} \mathrm{~T}^{3}\right)$

$74.8 \mathrm{~kJ} / \mathrm{mol}$

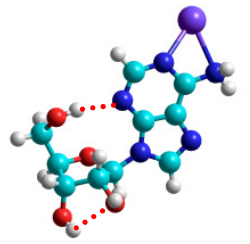

$\mathrm{B} 9\left(\mathrm{~N} 1 \mathrm{NH}_{2}\right)$

syn, $\mathrm{C} 2$ '-endo $\left({ }^{2} \mathrm{~T}_{1}\right)$

$77.2 \mathrm{~kJ} / \mathrm{mol}$

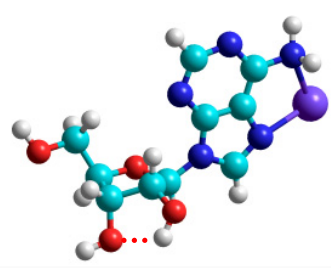

$\mathrm{B} 6\left(\mathrm{~N}_{7} \mathrm{NH}_{2}\right)$

syn, $\mathrm{C} 2$ '-endo $\left({ }^{2} \mathrm{~T}_{1}\right)$

$68.9 \mathrm{~kJ} / \mathrm{mol}$

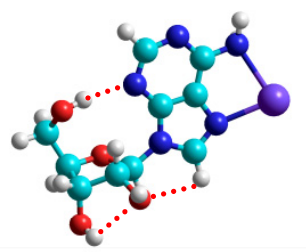

$\mathrm{B} 10\left(\mathrm{~N} 7 \mathrm{NH}_{2}\right)$

syn, $\mathrm{C} 2$ '-endo $\left({ }^{2} \mathrm{E}\right)$

$69.8 \mathrm{~kJ} / \mathrm{mol}$

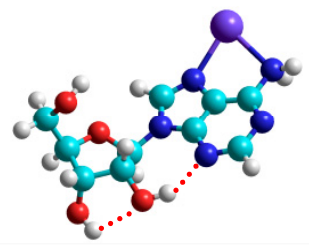

$\mathrm{B} 12\left(\mathrm{~N} \mathrm{NH}_{2}\right)$

anti, $\mathrm{C} 2$ '-endo $\left({ }^{2} \mathrm{~T}_{1}\right)$

$72.1 \mathrm{~kJ} / \mathrm{mol}$

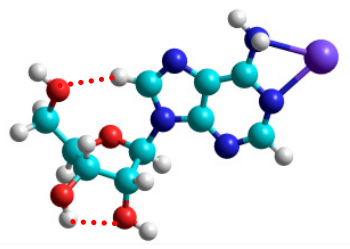

$\mathrm{B} 7\left(\mathrm{~N} 1 \mathrm{NH}_{2}\right)$

anti, $\mathrm{C} 3$ '-endo $\left({ }^{3} \mathrm{~T}_{2}\right)$

$74.9 \mathrm{~kJ} / \mathrm{mol}$

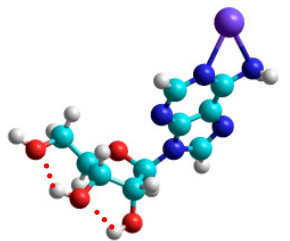

$\mathrm{B} 10\left(\mathrm{~N} 1 \mathrm{NH}_{2}\right)$

syn, C4'-exo $\left({ }_{4} \mathrm{~T}^{3}\right)$

$77.3 \mathrm{~kJ} / \mathrm{mol}$ 
Figure S3.

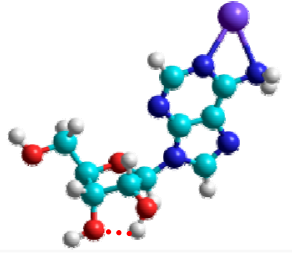

$\mathrm{B} 11\left(\mathrm{~N} 1 \mathrm{NH}_{2}\right)$

syn, $\mathrm{C} 2$ '-endo $\left({ }^{2} \mathrm{~T}_{1}\right)$

$78.0 \mathrm{~kJ} / \mathrm{mol}$

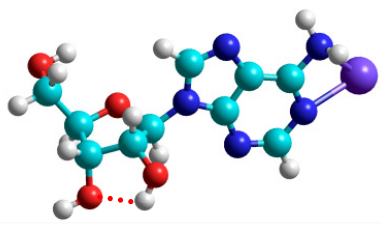

B12 $\left(\mathrm{N} \mathrm{NH}_{2}\right)$ anti, C1'-exo $\left({ }_{1} \mathrm{~T}^{2}\right)$ $80.0 \mathrm{~kJ} / \mathrm{mol}$

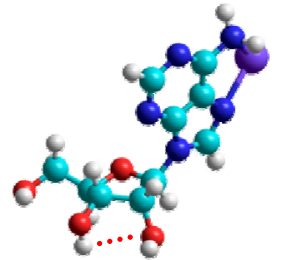

$\mathrm{B} 17\left(\mathrm{~N} \mathrm{NH}_{2}\right)$ syn, C4'-exo $\left({ }_{4} \mathrm{~T}^{3}\right)$ $83.8 \mathrm{~kJ} / \mathrm{mol}$

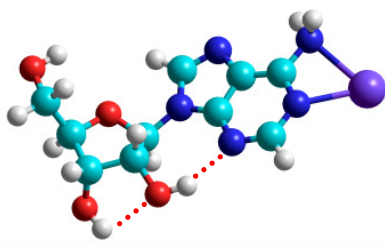

B16 $\left(\mathrm{N} \mathrm{NH}_{2}\right)$ anti, C2'-endo $\left({ }^{2} \mathrm{~T}_{1}\right)$ $88.3 \mathrm{~kJ} / \mathrm{mol}$

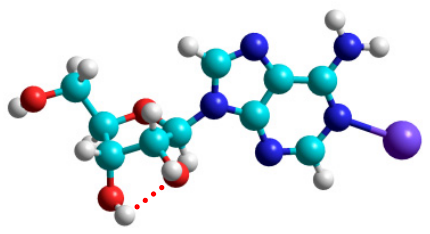

$\mathrm{M} 1$ (N1)

anti, C2'-endo $\left({ }^{2} \mathrm{~T}_{1}\right)$ $96.1 \mathrm{~kJ} / \mathrm{mol}$

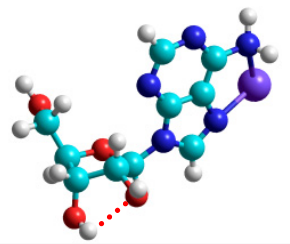

B13 $\left(\mathrm{N} \mathrm{NH}_{2}\right)$

syn, C2'-endo $\left({ }^{2} \mathrm{~T}_{1}\right)$

$79.5 \mathrm{~kJ} / \mathrm{mol}$

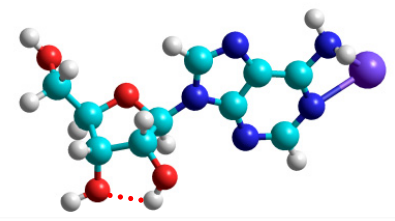

$\mathrm{B} 13\left(\mathrm{~N} 1 \mathrm{NH}_{2}\right)$

anti, $\mathrm{C} 1$ '-exo $\left({ }_{1} \mathrm{~T}^{2}\right)$

$81.5 \mathrm{~kJ} / \mathrm{mol}$

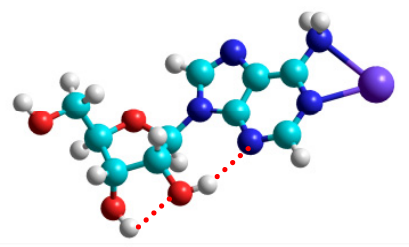

$\mathrm{B} 14\left(\mathrm{~N} 1 \mathrm{NH}_{2}\right)$

anti, C2'-endo $\left({ }^{2} \mathrm{E}\right)$

$85.3 \mathrm{~kJ} / \mathrm{mol}$

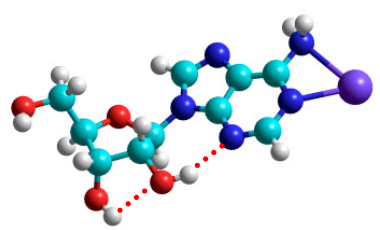

$\mathrm{B} 17\left(\mathrm{~N} 1 \mathrm{NH}_{2}\right)$ anti, C2'-endo $\left({ }^{2} \mathrm{~T}_{1}\right)$ $90.6 \mathrm{~kJ} / \mathrm{mol}$

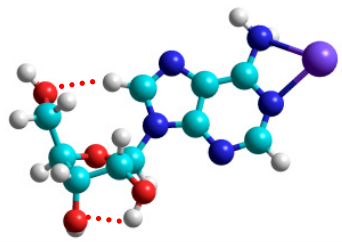

$\mathrm{B} 20\left(\mathrm{~N} 1 \mathrm{NH}_{2}\right)$

anti, C3'-exo $\left({ }_{3} \mathrm{~T}^{4}\right)$

$96.3 \mathrm{~kJ} / \mathrm{mol}$

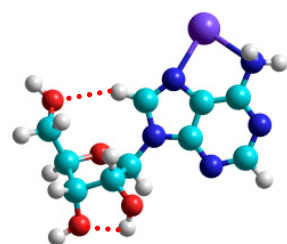

B14 $\left(\mathrm{N} \mathrm{NH}_{2}\right)$

anti, C3'-exo $\left({ }_{3} \mathrm{~T}^{2}\right)$

$79.6 \mathrm{~kJ} / \mathrm{mol}$

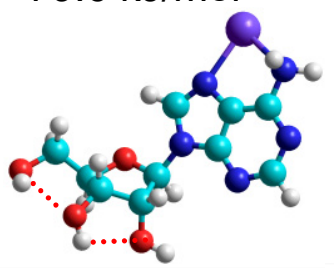

$\mathrm{B} 16\left(\mathrm{~N} \mathrm{NH}_{2}\right)$

anti, C3'-endo $\left({ }_{4}{ }^{3} \mathrm{~T}\right)$

$82.3 \mathrm{~kJ} / \mathrm{mol}$

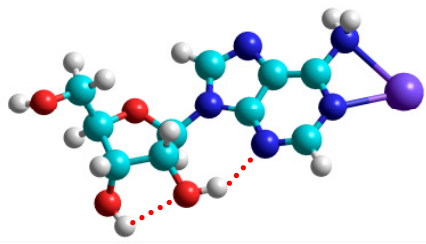

$\mathrm{B} 15\left(\mathrm{~N} 1 \mathrm{NH}_{2}\right)$

anti, $\mathrm{C} 2$ '-endo $\left({ }^{2} \mathrm{E}\right)$

$85.6 \mathrm{~kJ} / \mathrm{mol}$

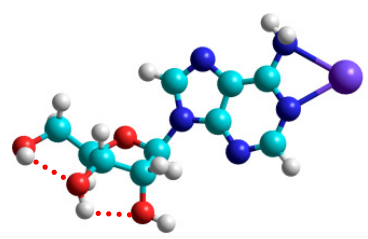

$\mathrm{B} 18\left(\mathrm{~N} 1 \mathrm{NH}_{2}\right)$

anti, C3'-endo $\left({ }_{4}{ }^{3 \mathrm{~T}}\right)$

$91.3 \mathrm{~kJ} / \mathrm{mol}$

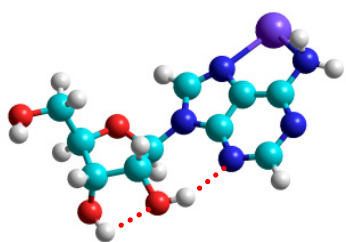

B15 $\left(\mathrm{N} \mathrm{NH}_{2}\right)$

anti, C2'-endo $\left({ }^{2} \mathrm{~T}_{1}\right)$

$79.7 \mathrm{~kJ} / \mathrm{mol}$

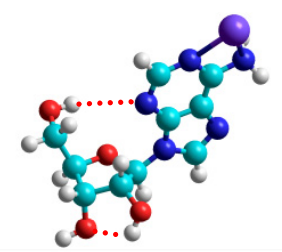

B13 $\left(\mathrm{N} \mathrm{NH}_{2}\right)$

syn, C2'-endo $\left({ }^{2} \mathrm{~T}_{1}\right)$

$82.6 \mathrm{~kJ} / \mathrm{mol}$

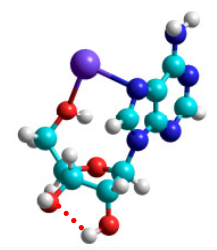

B1(N7O5')

syn, C2'-exo $\left({ }_{2} \mathrm{E}\right)$

$86.1 \mathrm{~kJ} / \mathrm{mol}$

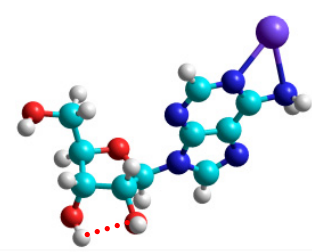

$\mathrm{B} 19\left(\mathrm{~N} 1 \mathrm{NH}_{2}\right)$

syn, C2'-endo $\left({ }^{2} \mathrm{~T}_{1}\right)$

$92.5 \mathrm{~kJ} / \mathrm{mol}$ 
Figure S4.

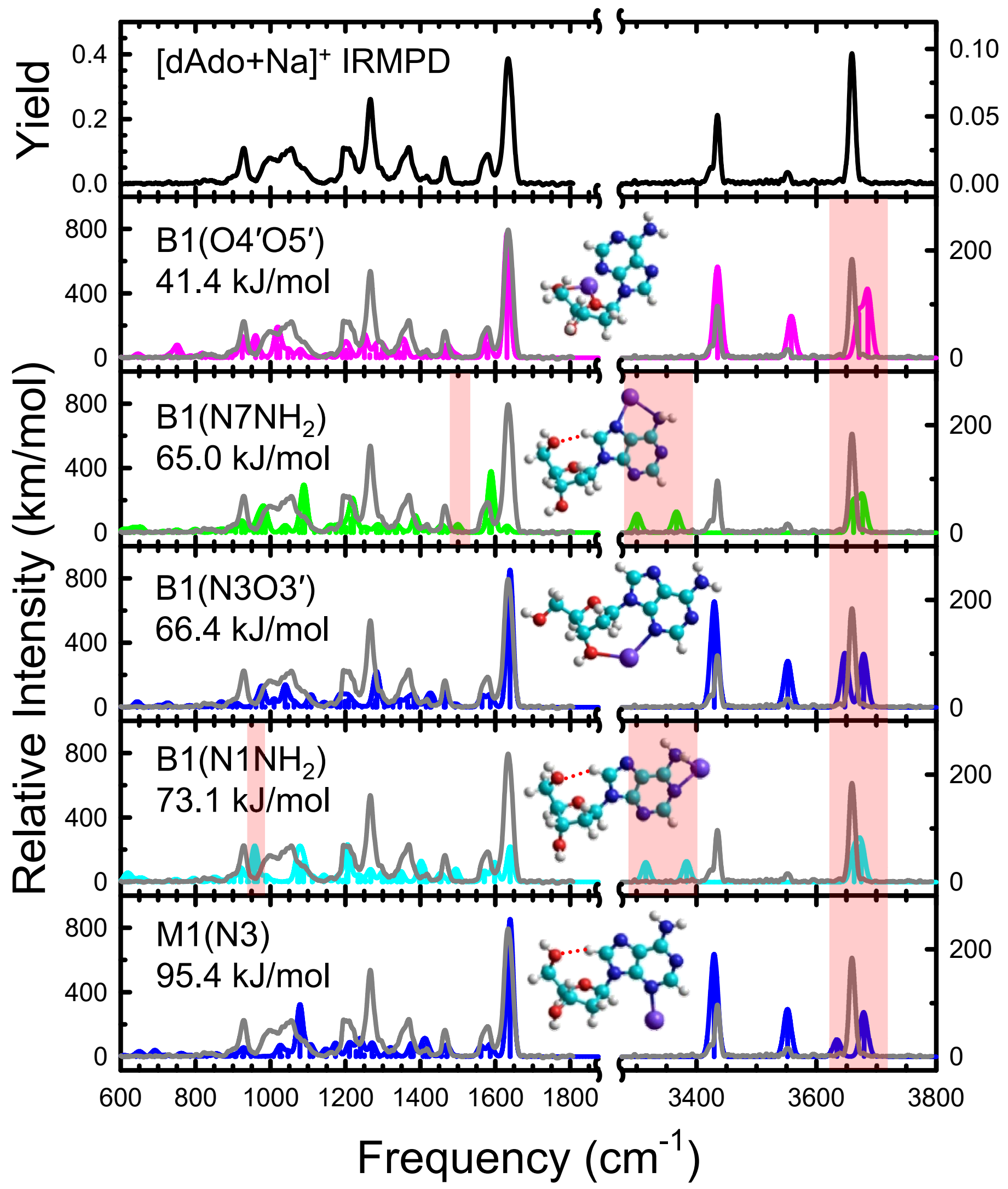


Figure S5.

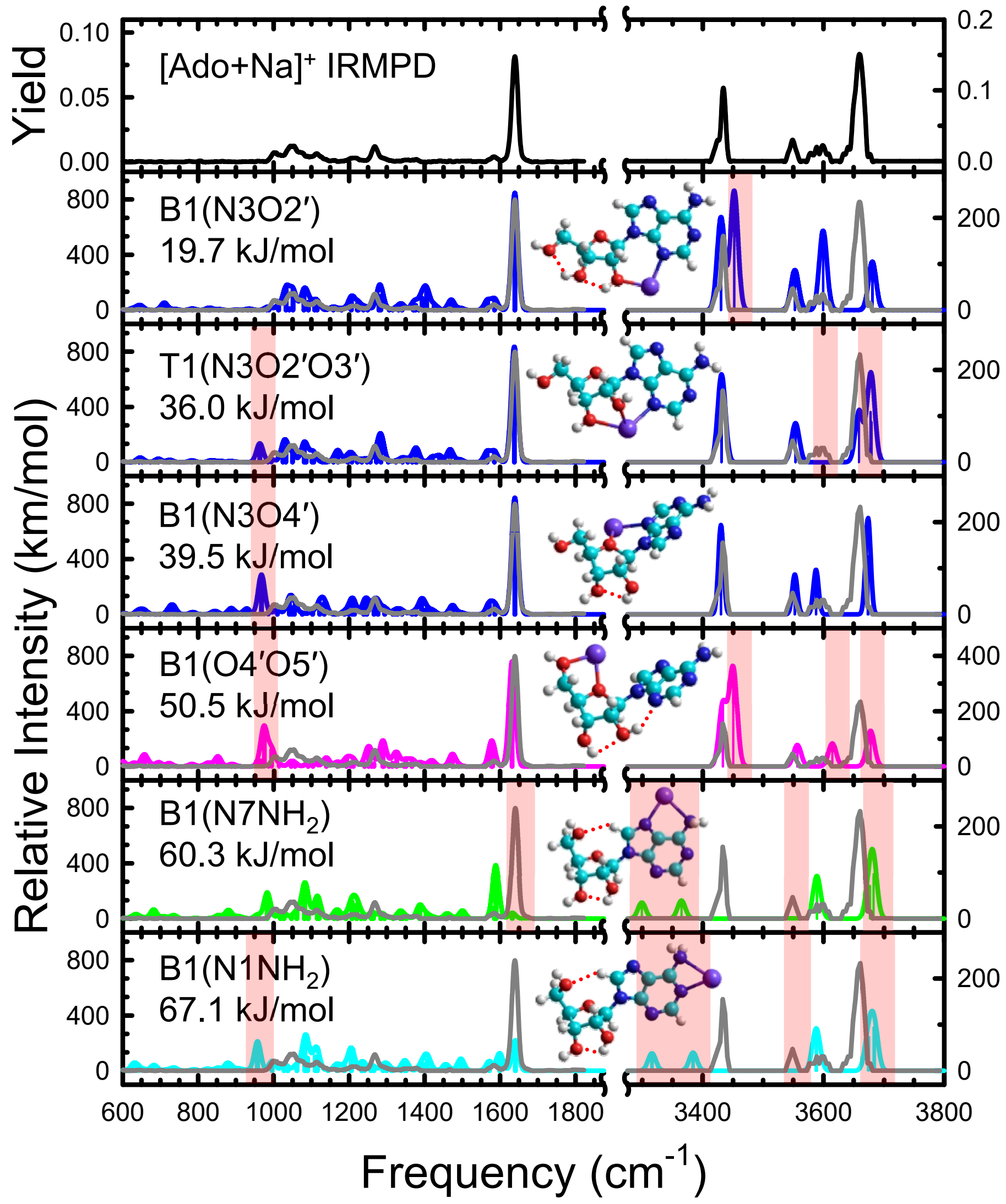

